\title{
Recombinant annexin $A 6$ promotes membrane repair and protects against muscle injury
}

\author{
Alexis R. Demonbreun,, ${ }^{1,2}$ Katherine S. Fallon, ${ }^{1}$ Claire C. Oosterbaan, ${ }^{1}$ Elena Bogdanovic, ${ }^{1}$ James L. Warner, ${ }^{1}$ Jordan J. Sell, ${ }^{1}$ \\ Patrick G. Page, ${ }^{1}$ Mattia Quattrocelli, ${ }^{1}$ David Y. Barefield, ${ }^{1}$ and Elizabeth M. McNally'
}

'Center for Cenetic Medicine, and 2Department of Pharmacology, Northwestern University, Chicago, Illinois, USA.

\begin{abstract}
Membrane repair is essential to cell survival. In skeletal muscle, injury often associates with plasma membrane disruption. Additionally, muscular dystrophy is linked to mutations in genes that produce fragile membranes or reduce membrane repair. Methods to enhance repair and reduce susceptibility to injury could benefit muscle in both acute and chronic injury settings. Annexins are a family of membrane-associated $\mathrm{Ca}^{2+}$-binding proteins implicated in repair, and annexin $\mathrm{A} 6$ was previously identified as a genetic modifier of muscle injury and disease. Annexin A6 forms the repair cap over the site of membrane disruption. To elucidate how annexins facilitate repair, we visualized annexin cap formation during injury. We found that annexin cap size positively correlated with increasing $\mathrm{Ca}^{2+}$ concentrations. We also found that annexin overexpression promoted external blebs enriched in $\mathrm{Ca}^{2+}$ and correlated with a reduction of intracellular $\mathrm{Ca}^{2+}$ at the injury site. Annexin $\mathrm{A} 6$ overexpression reduced membrane injury, consistent with enhanced repair. Treatment with recombinant annexin A6 protected against acute muscle injury in vitro and in vivo. Moreover, administration of recombinant annexin A6 in a model of muscular dystrophy reduced serum creatinine kinase, a biomarker of disease. These data identify annexins as mediators of membrane-associated $\mathrm{Ca}^{2+}$ release during membrane repair and annexin $\mathrm{A6}$ as a therapeutic target to enhance membrane repair capacity.
\end{abstract}

\section{Introduction}

Plasma membrane repair occurs after membrane disruption and is a highly conserved process. The active process required for resealing membrane disruptions is thought to rely on $\mathrm{Ca}^{2+}$-dependent vesicle fusion and local cytoskeletal remodeling $(1,2)$. Other models suggest that membrane repair is mediated through the fusion of lysosomal vesicles, lateral diffusion of membrane to the site of injury, and the extrusion of membranous blebs (3-7). These models are not mutually exclusive and may depend on the type and extent of damage. Skeletal muscle is highly dependent on plasma membrane repair, as mutations in genes encoding repair proteins lead to muscle disease (8-13).

The annexins are a family of $\mathrm{Ca}^{2+}$-binding proteins that regulate lipid binding, cytoskeletal reorganization, and membrane folding, steps necessary for membrane repair (14-19). Individual annexin-repeat domains coordinate $\mathrm{Ca}^{2+}$ binding with unique annexin-specific type II or type III binding sites. Differential $\mathrm{Ca}^{2+}$ affinity of the type II and type III binding sites provides each annexin a unique ability to respond to a range of intracellular $\mathrm{Ca}^{2+}$ levels and phospholipid binding (20). Annexins have the ability to self- and hetero-oligomerize (21). Typical annexins like A1 and A2 contain 1 annexin core composed of 4 annexin-repeat domains. In contrast, annexin A6 contains 2 annexin cores and thus 8 annex-

Conflict of interest: Northwestern University filed a provisional patent (number $62 / 783,619$ ) on behalf of the authors (ARD and EMM) related to the content of this work. Copyright: () 2019, American Society for Clinical Investigation.

Submitted: March 14, 2019; Accepted: July 26, 2019; Published: September 23, 2019. Reference information: / Clin Invest. 2019;129(11):4657-4670,

https://doi.org/10.1172/JCl128840. in-repeat domains (22). Annexin A6's duplicated structure makes it possible for the amino- and carboxyl-terminal annexin core domains to bind 1 or 2 distinct membranes, making annexin A6 a prime target for facilitating membrane coalescence and folding required during membrane repair $(18,19,23)$.

Annexins have a high affinity for phosphatidylserine, phosphatidylinositol, and cholesterol, which are highly enriched in the sarcolemma $(24,25)$. Multiple annexins, including annexins A1, A2, and A6, have been implicated in membrane repair in skeletal muscle, as well as Xenopus oocytes, human trophoblasts, and HeLa cancer cells, suggesting a conserved mechanism (5, 26-32). Annexins are recruited to the injured membrane in a sequential manner, forming a macromolecular repair complex at the membrane lesion referred to as a repair cap $(5,19,29)$. We previously identified a polymorphism in Anxa6, the gene encoding annexin A6, in several commonly used experimental mouse strains that correlated with impaired muscle repair (33-35). This polymorphism produces a truncated annexin A6 protein that acts in a dominant-negative manner to reduce repair cap formation and interferes with sarcolemmal repair. Additional studies in mice have shown that loss of annexin A2 results in poor myofiber repair (12). These data suggest that there is a coordinated recruitment of annexin proteins to the repair cap facilitated by dynamic protein-protein interactions.

Here, we assessed the kinetics of annexin A1, A2, and A6 in repair cap formation after membrane injury at multiple $\mathrm{Ca}^{2+}$ concentrations. The repair cap formed by annexins A1, A2, and $\mathrm{A} 6$ increased with increasing $\mathrm{Ca}^{2+}$ concentrations, while mutations in $\mathrm{Ca}^{2+}$-coordinating residues interfered with normal annexin repair cap formation. Annexin overexpression promoted the formation of external blebs at the site of mem- 
A

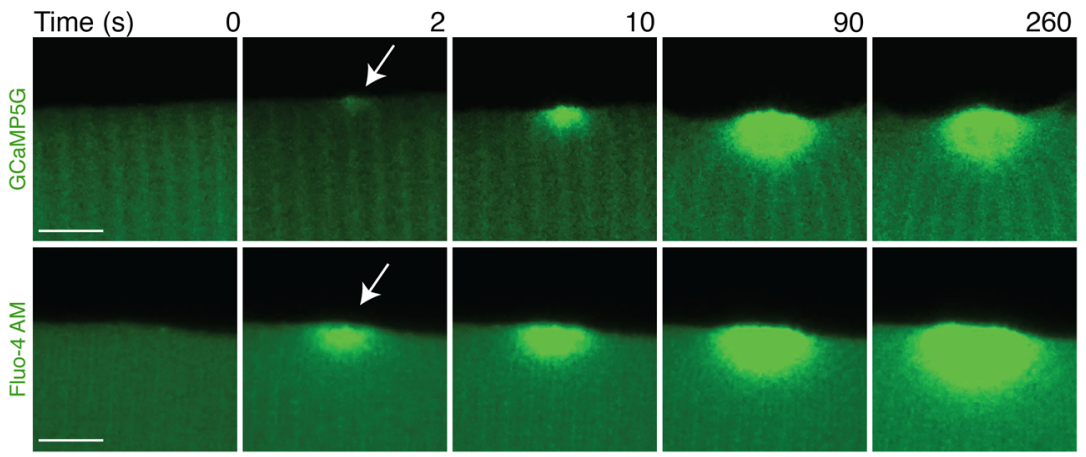

B
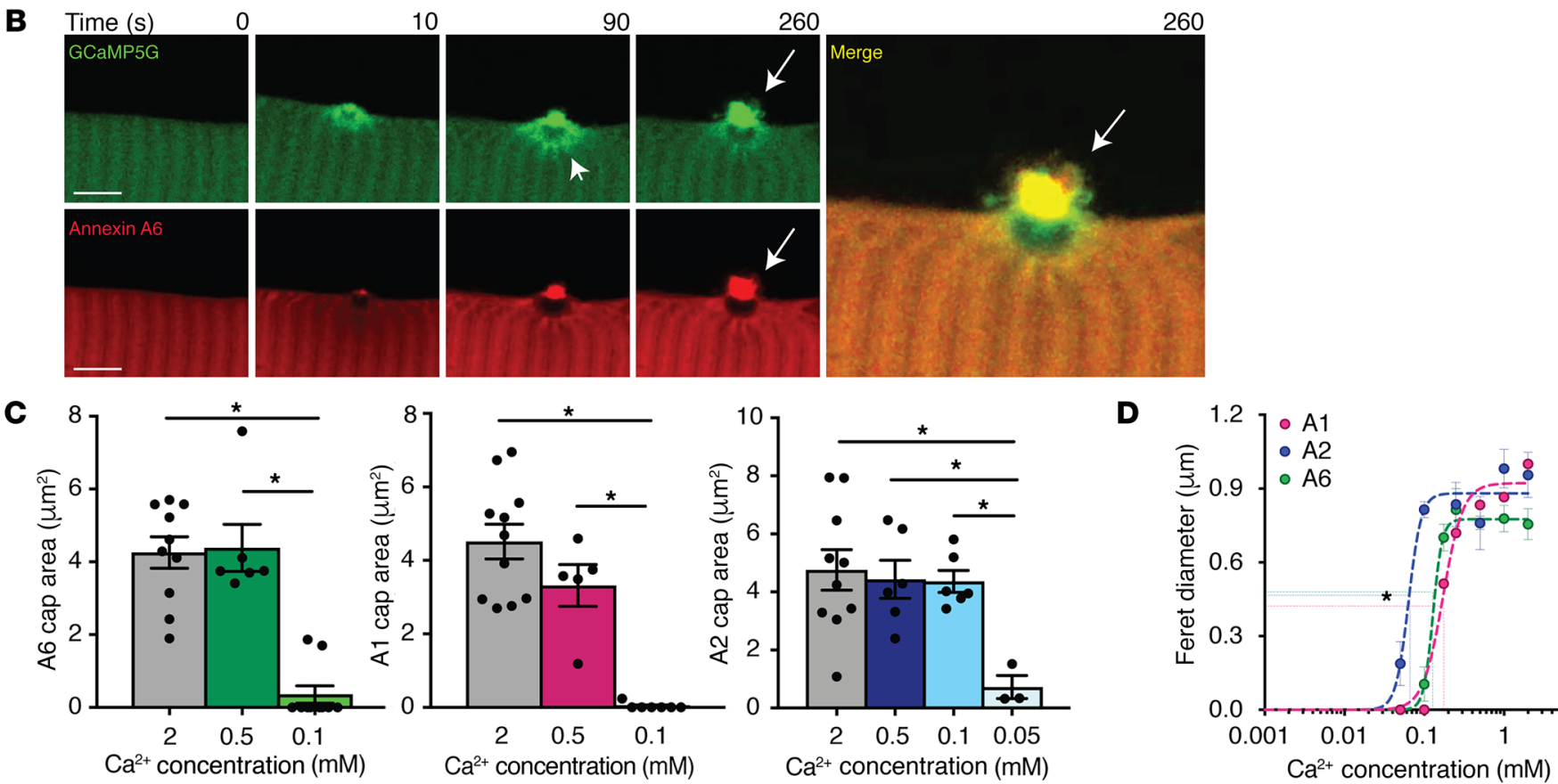

Figure 1. $\mathrm{Ca}^{2+}$-dependent annexin repair cap recruitment at the site of injury. Myofibers were generated to express the Ca ${ }^{2+}$ indicator $\mathrm{GCaMP5G}$ (green), and time-lapse single-slice images were assessed at time points after membrane disruption. (A) GCaMP5C fluorescence was present at the site of injury, at 2 seconds (arrow), indicating the presence of $\mathrm{Ca}^{2+}$ immediately after damage at the site of injury (top panel). These data were validated with a non-protein-based $\mathrm{Ca}^{2+}$ indicator, Fluo-4 AM (green, bottom panel). (B) Time-lapse images of myofibers coelectroporated with GCaMP5C and annexin A6-tdTomato (A6, red). GCaMP5G fluorescence was present at the site of injury localized around the annexin A6-free zone (arrowhead) and at the annexin A6 cap (arrow). GCaMP5G colocalized (merge, yellow, arrow) with the annexin A6 repair cap. Scale bars: $5 \mu \mathrm{m}$. (C) Myofibers expressing fluorescently tagged annexins A1, A2, or A6 were injured at multiple $\mathrm{Ca}^{2+}$ concentrations. Annexin $\mathrm{A} 1$ and $\mathrm{A} 6$ repair cap size was reduced at $0.1 \mathrm{mM} \mathrm{Ca}^{2+}$ compared with $2 \mathrm{mM}$ and $0.5 \mathrm{mM}$. Annexin $\mathrm{A} 2$ repair cap area was significantly reduced at $0.05 \mathrm{mM} \mathrm{Ca}^{2+}$ compared with $2 \mathrm{mM}, 0.5 \mathrm{mM}$, and $0.1 \mathrm{mM} \mathrm{Ca}^{2+}$. (D) Cap kinetics were plotted as cap Feret diameter over a range of $\mathrm{Ca}^{2+}$ concentrations. Annexin $\mathrm{A} 2$ had a statistically significant leftward shift in $K_{m}(1 / 2)$, followed by annexin $A 6$ and then $A 1$. Data are expressed as mean \pm SEM. Differences were tested by 1-way ANOVA with Tukey's multiple-comparisons test (C). ${ }^{*} P<0.05$ ( $n=5$ myofibers per condition).

brane injury that were released from the repair cap. Overexpression of annexin A6 resulted in the formation of larger blebs being released from the repair cap. These vesicles were enriched in $\mathrm{Ca}^{2+}$-indicator protein GCaMP5G, and this enrichment of $\mathrm{Ca}^{2+}$ correlated with a reduction of intracellular $\mathrm{Ca}^{2+}$ fluorescence near the injury site. Annexin A6 overexpression promoted membrane repair, while mutation of residue E233, a critical $\mathrm{Ca}^{2+}$-coordinating residue in annexin $\mathrm{A} 6$, interfered with annexin repair-complex formation and decreased repair capacity. Local and systemic administration of recombinant annexin A6 reduced muscle damage in vivo. These data identify a role for annexins in bleb release from muscle membrane lesions during membrane repair and identify annexin A6 as a therapeutic target to protect against muscle injury.

\section{Results}

$\mathrm{Ca}^{2+}$ localizes to the repair cap upon membrane damage. Activation of muscle membrane repair requires the presence of external $\mathrm{Ca}^{2+}$ (8). We previously showed that annexin proteins aggregate into repair caps at the site of injury bordered by an annexin-free zone within the cytoplasm under the repair cap $(5,33,35,36)$. To visualize $\mathrm{Ca}^{2+}$ dynamics at the site of injury in real time, we utilized an in vivo fluorescent $\mathrm{Ca}^{2+}$ indicator protein, GCaMP5G. GCaMP5G is a fusion protein composed of green fluorescent protein (GFP), the calcium-binding protein calmodulin, and the calmodulin M13 binding peptide. GCaMP5G has minimal fluorescence when not bound to $\mathrm{Ca}^{2+}$, and $\mathrm{Ca}^{2+}$ binding results in a conformational change within the protein, increasing the fluorescence intensity of GFP (37). We electroporated wild-type flexor digitorum brevis 

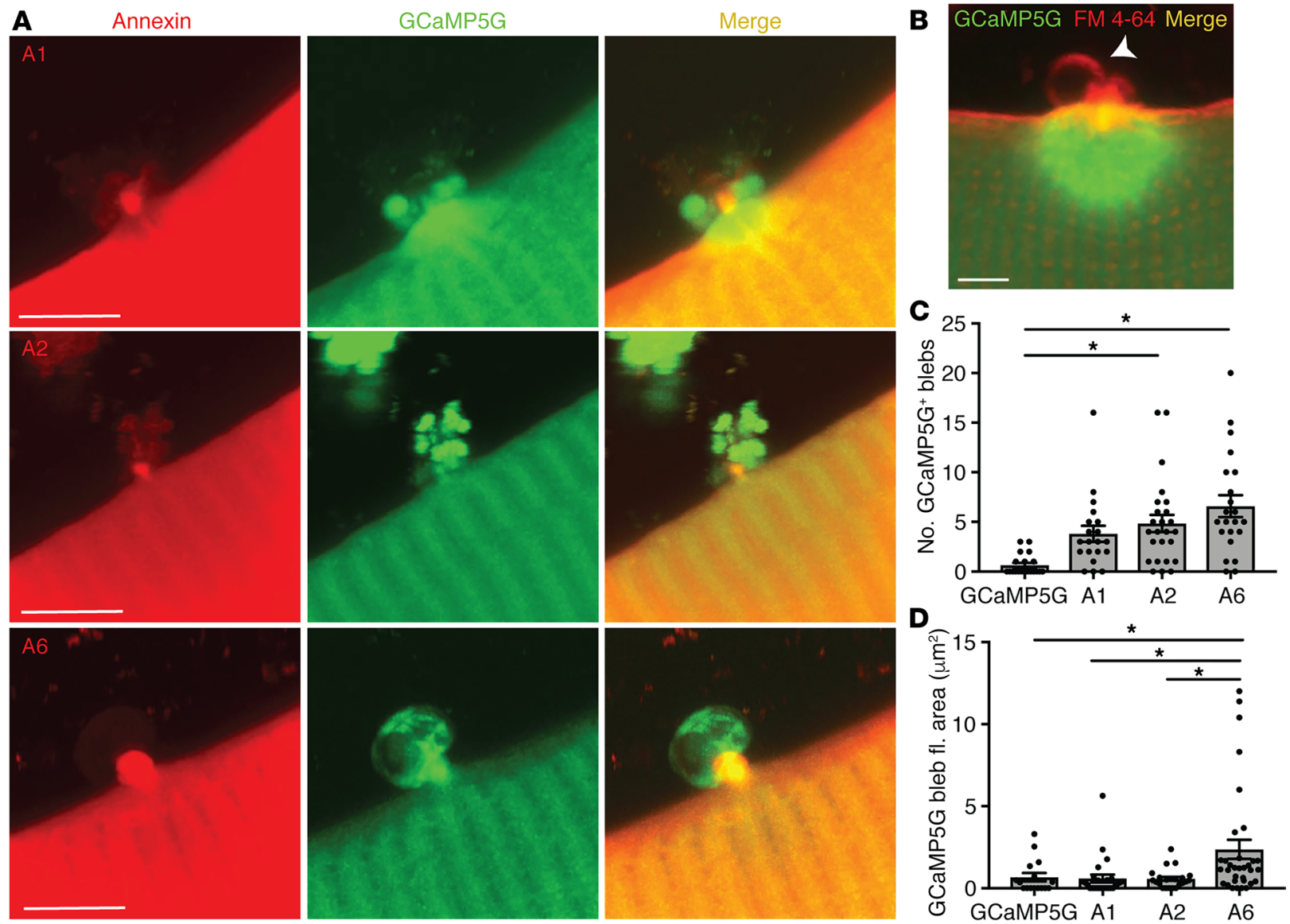

Figure 2. Annexin expression promoted release of blebs from the site if myofiber repair. Myofibers were electroporated with the Ca2+ indicator GCaMP5 $\mathrm{C}$ (green) with or without tdTomato-labeled annexin A1, annexin A2, or annexin A6. Ca ${ }^{2+}$ area and fluorescence were assessed after membrane damage. (A) High-magnification Z-projection images illustrate external blebs filled with the $\mathrm{Ca}^{2+}$ indicator emanating from the lesion when annexin $\mathrm{A} 1$, $\mathrm{A2}$, or $\mathrm{A} 6$ was coexpressed and a corresponding reduction in $\mathrm{Ca}^{2+}$ indicator within the myofiber when compared with GCaMP5C alone (see panel B). (B) Membrane marked by FM 4-64 shows GCaMP5G-negative vesicles form in the absence of annexin overexpression. Scale bars: $5 \mu \mathrm{m}$. (C) Expression of annexin A6 or A2 resulted in an increased number of GCaMP5G-positive blebs. (D) Expression of annexin A6 resulted in the formation of the largest GCaMP5G-positive blebs. Data are expressed as mean \pm SEM. Differences were tested by 1-way ANOVA with Tukey's multiple-comparisons test. ${ }^{*} P<0.05(n=16$ myofibers from $n=3$ mice per condition).

(FDB) muscle with the GCaMP5G plasmid and then injured the plasma membrane using laser ablation $(5,38)$. Within 2 seconds of membrane injury (arrow in Figure 1A), GCaMP5G fluorescence accumulated in the cytoplasm at the site of injury. GCaMP5G fluorescence intensity progressively increased through 260 seconds of imaging (Figure 1A).

To ensure these results were not a reflection of protein aggregation of the GCaMP5G sensor, we injured wild-type myofibers in the presence of Fluo-4 AM. Fluo-4 AM is a non-protein-based, $\mathrm{Ca}^{2+}$ indicator dye that increases fluorescence intensity upon binding $\mathrm{Ca}^{2+}$ and is routinely used to measure $\mathrm{Ca}^{2+}$ dynamics. Similarly to GCaMP5G fluorescence, Fluo-4 AM fluorescence intensity increased at the site of laser-induced membrane injury 2 seconds after damage (arrow) and continued to increase intensity through the 260 seconds of imaging (Figure 1A). In myofibers coelectroporated with plasmids expressing GCaMP5G and annexin A6 with a carboxyl-terminal tdTomato fluorescent tag, GCaMP5G fluorescence localized in a ring around the annexin A6-free zone (Figure $1 \mathrm{~B}$, arrowhead) and colocalized with annexin A6 at the repair cap
(Figure 1B, arrow, merge). We also evaluated whether $\mathrm{pH}$ changed with injury using pHrodo fluorescence, a non-protein-based $\mathrm{pH}$ indicator dye that changes fluorescence with different $\mathrm{pH}$ levels. We noted no change from the preinjury state (0 seconds) compared with 10 seconds after injury, when $\mathrm{Ca}^{2+}$ indicator fluorescence is already increased at the site of injury (Supplemental Figure 1; supplemental material available online with this article; https://doi.org/10.1172/JCI128840DS1). This temporal sequence is consistent with $\mathrm{Ca}^{2+}$ accumulation at the site of injury facilitating annexin translocation and assembly into repair caps.

Annexin repair caps exhibit differential $\mathrm{Ca}^{2+}$ sensitivity during repair cap recruitment. Annexin proteins are $\mathrm{Ca}^{2+}$-dependent phospholipid- and actin-binding proteins that contain 4 annexinrepeat domains, or 8 in the case of annexin A6 (Supplemental Figure 2). Annexin-repeat domains bind $\mathrm{Ca}^{2+}$, but are distinct from the $\mathrm{Ca}^{2+}$ binding of $\mathrm{C} 2$ domains and EF-hands seen in other classes of repair proteins (39). Annexins coordinate $\mathrm{Ca}^{2+}$ and bind membranes from their convex face (Supplemental Figure 2), and both type II and type III $\mathrm{Ca}^{2+}$-binding sites have been described 


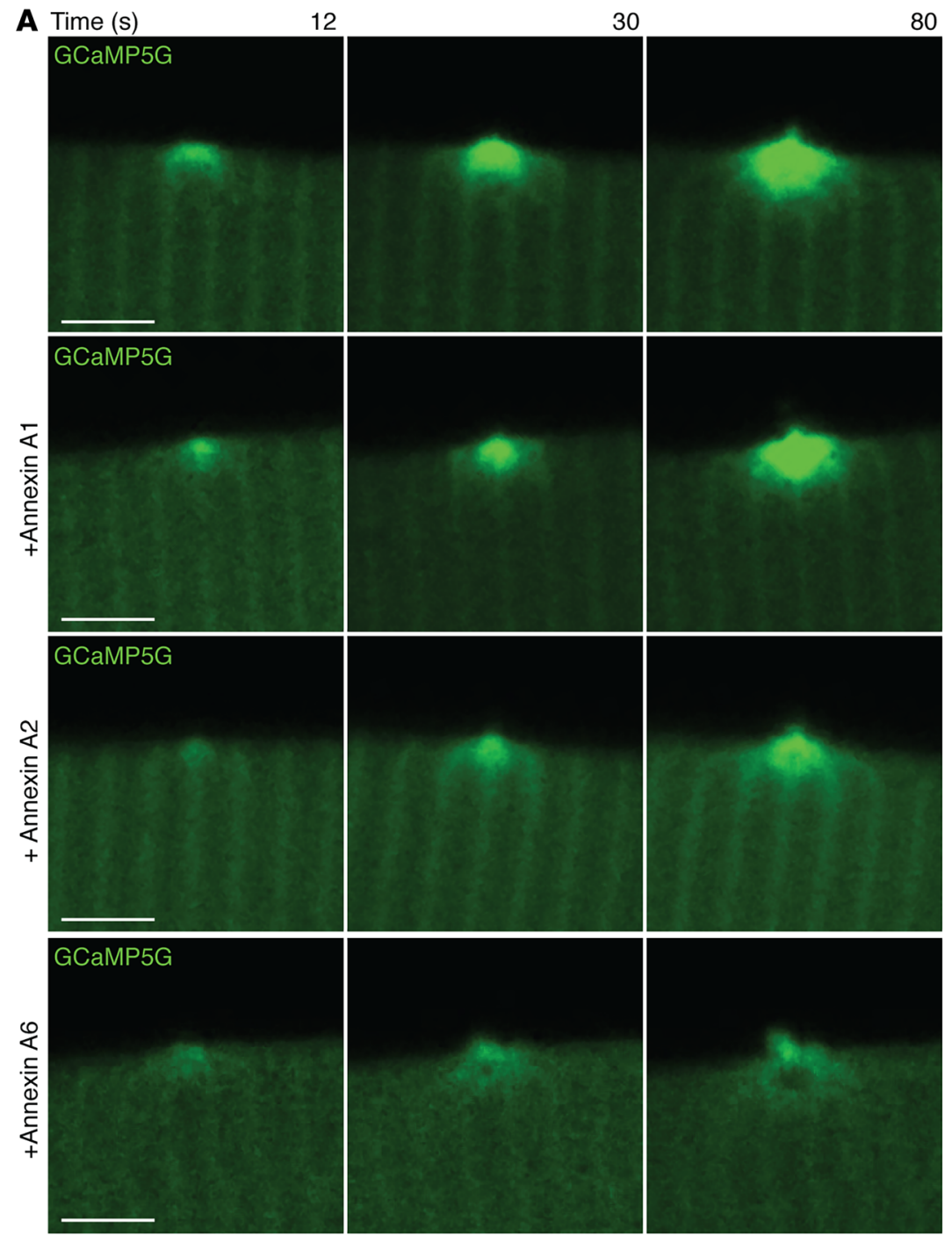

B
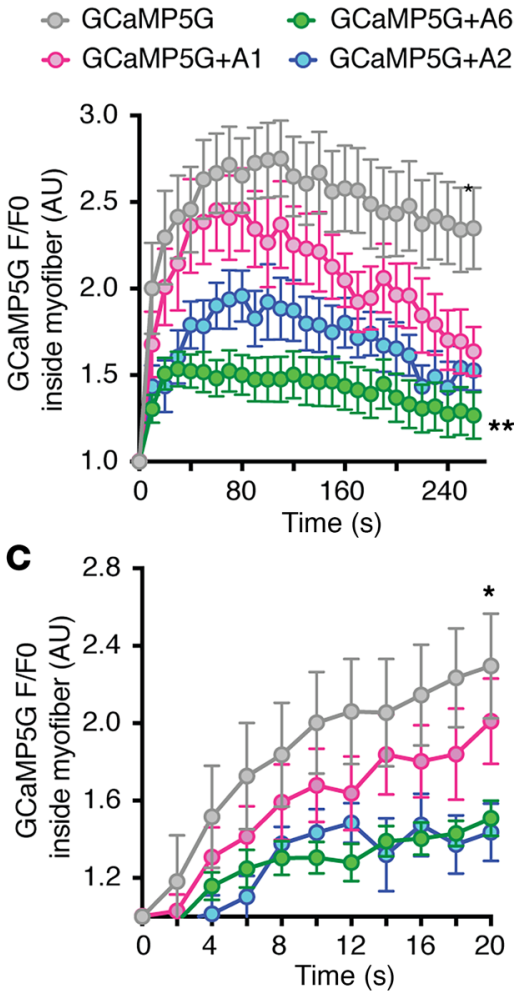

D

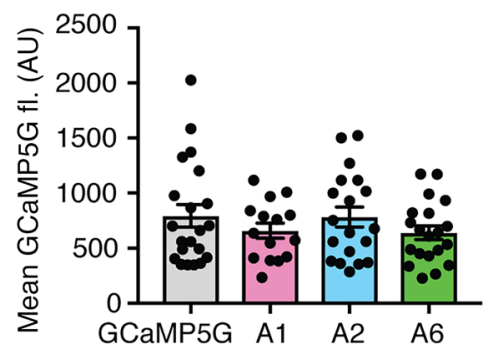

Figure 3. Annexin expression reduced $\mathrm{Ca}^{2+}$ within the myofiber. Myofibers were electroporated with the $\mathrm{Ca}^{2+}$ indicator GCaMP5G (green) with or without tdTomato-labeled annexin A1, annexin A2, or annexin A6 (red imaging not shown in this image). $\mathrm{Ca}^{2+}$ area and fluorescence were assessed after membrane damage. (A) Time-lapse single-slice images reveal that coexpression of either annexin A1, A2, or A6 resulted in a significant reduction in GCaMP5G fluorescence (green) measured inside the myofiber at the site of injury over time. (B) Expression of either annexin A1, A2, or A6 resulted in a significant reduction in GCaMP5C fluorescence measured inside the myofiber at the site of injury over 240 seconds of imaging, with annexin A6 inducing the greatest reduction in GCaMP5C fluorescence. (C) Both annexin A2 and A6 contributed to the early reduction in GCaMP5C fluorescence, as seen by imaging during the first 20 seconds after injury. (D) Initial GCaMP5G mean fluorescence was not significantly different between groups. Scale bars: $5 \mu \mathrm{m}$. Data are expressed as mean \pm SEM. Differences were tested by 2-way ANOVA with Bonferroni's multiple-comparisons test (B and C) or 1-way ANOVA with Tukey's multiple-comparisons test (D). ${ }^{*} P<0.05$ ( $n=9$ myofibers from $n=3$ mice per condition).

in annexin proteins. To further define the $\mathrm{Ca}^{2+}$ requirements in annexin-mediated sarcolemmal repair in myofibers, we examined annexin $\mathrm{A} 1, \mathrm{~A} 2$, or $\mathrm{A} 6$ repair cap formation at multiple $\mathrm{Ca}^{2+}$ concentrations. Cap size was measured from the center of a $Z$-stack, and the type of fluorescent tag, turboGFP or tdTomato, did not alter assessed parameters (Supplemental Figure 3, A and B). Annexin $\mathrm{A} 1$ and $\mathrm{A} 6$ repair cap size was $\mathrm{Ca}^{2+}$ dependent, with the largest repair caps forming at $2 \mathrm{mM}$ and smaller repair caps forming at 0.1 $\mathrm{mM}$, while annexin A2 repair caps were not significantly reduced until $0.05 \mathrm{mM} \mathrm{Ca}^{2+}$ (Figure 1, C and D). Repair cap area was plotted as a function of $\mathrm{Ca}^{2+}$ concentration using a modified Hill equation. Annexin A2 formed a repair cap at the lowest concentration of $\mathrm{Ca}^{2+}(0.05 \mathrm{mM})$, while annexins $\mathrm{A} 1$ and A6 did not form a dis- cernable cap at $\mathrm{Ca}^{2+}$ concentrations lower than $0.1 \mathrm{mM}$, seen as the significantly left-shifted annexin A2 curve with a $K_{m}(1 / 2)$ of $0.067 \mathrm{mM}$ compared with A6 and A1, which showed a $K_{m}(1 / 2)$ of $0.12 \mathrm{mM}$ and $0.17 \mathrm{mM}$, respectively (Figure 1D). Annexin A1 and A6 repair cap size and formation rate were highly dependent on $\mathrm{Ca}^{2+}$ concentration (Supplemental Figure 4). The rate of annexin A2 cap formation and cap size was similar at $2 \mathrm{mM}, 0.5 \mathrm{mM}$, and $0.1 \mathrm{mM} \mathrm{Ca}^{2+}$, while annexin A1 and A6 rates decreased with lower $\mathrm{Ca}^{2+}$ concentrations, suggesting a high $\mathrm{Ca}^{2+}$ affinity for annexin A2 (Supplemental Figure 4). To ensure that repair cap formation was not an artifact due to the type of laser injury, we induced laser injury with both the Nikon A1R GaSP confocal and the Nikon A1R $\mathrm{MP}+$ multiphoton confocal. Injury induced by a multiphoton laser 

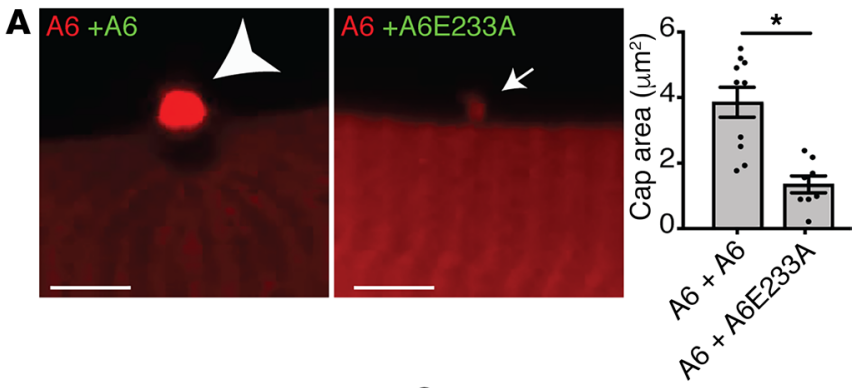

B

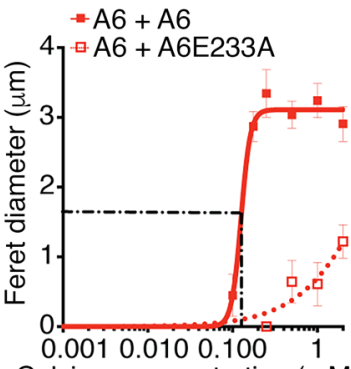

C
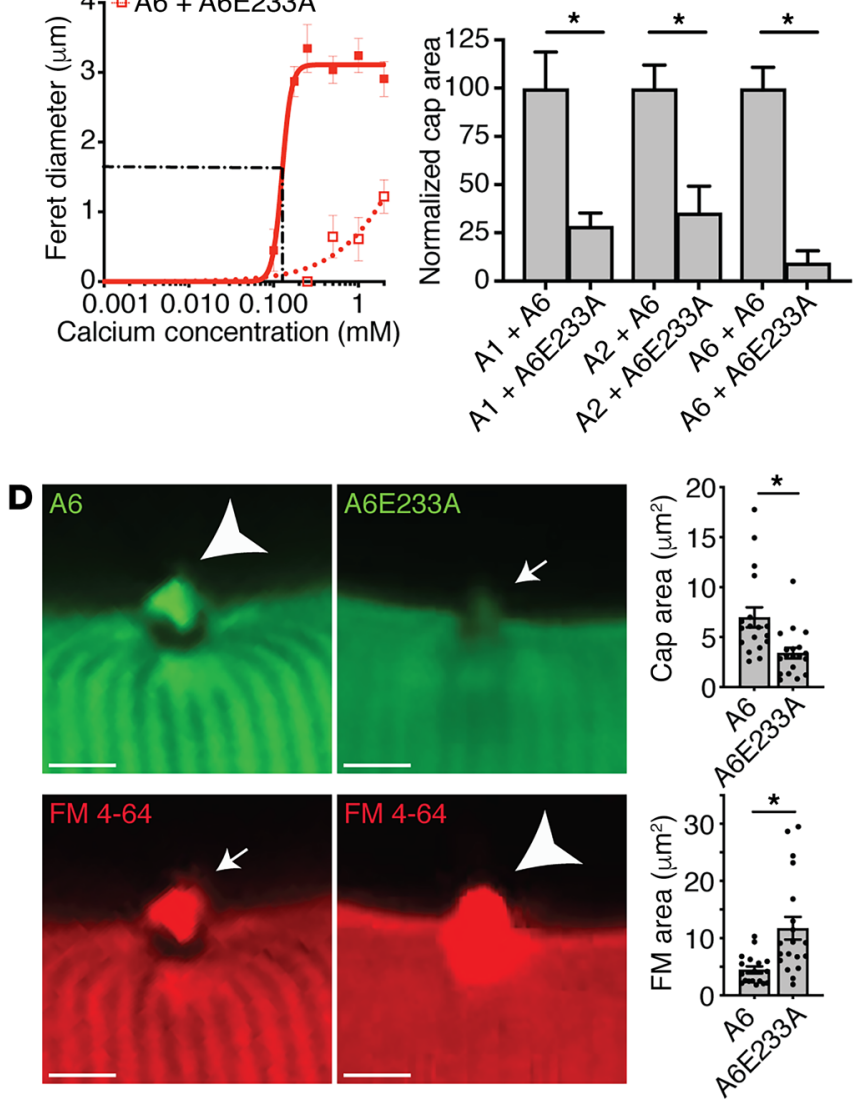

is more focused and produces less collateral damage. Annexin A6 repair caps appeared comparable with both types of lasers (Supplemental Figure 5). These data indicate that annexin A1, A2, and A6 repair cap formation is influenced by the level of $\mathrm{Ca}^{2+}$ present during myofiber repair, with annexin A2 being the most $\mathrm{Ca}^{2+}$ sensitive of the 3 annexins studied.

Annexin overexpression promotes bleb formation at the site of membrane injury. Membrane repair studies in Lytechinus pictus and Xenopus oocytes have observed membranous structures emerging and erupting from the site of membrane repair $(30,40)$. Additionally, in artificial membrane preparations, the addition of recombinant annexins induced membrane folding or blebbing in a $\mathrm{Ca}^{2+}$-dependent manner at sites of membrane imperfection $(18,19)$. We investigated whether similar findings could be observed at the site of muscle membrane injury in live skeletal myofibers. We expressed GCaMP5G alone or in combination with annexin A1, A2, or A6 in skeletal myofibers. We found that overexpression of annexins promoted the formation of extracellular blebs emanating from annexin repair caps at the
Figure 4. Annexin $\mathrm{A} 6 \mathrm{Ca}^{2+}$-binding mutant reduced annexin repair cap recruitment and decreased myofiber membrane repair capacity. (A) Myofibers were coelectroporated with wild-type-tdTomato (red labels) and either wild-type-GFP or mutant-GFP (green labels) annexin constructs, and cap size was assessed after membrane damage; only the red channel is shown to demonstrate the effect on wild-type annexin. (B) Coexpression of mutant annexin A6E233A was sufficient to reduce wild-type annexin A6 cap assembly. Cap kinetics were plotted as cap Feret diameter over a range of $\mathrm{Ca}^{2+}$ concentrations, from $0-2 \mathrm{mM}$. (C) Coexpression of annexin A6E233A was sufficient to significantly reduce the cap area of coexpressed annexin $\mathrm{A} 1, \mathrm{~A} 2$, and $\mathrm{A} 6 .{ }^{*} P<0.05$ for $\mathrm{WT}+\mathrm{WT}$ vs. WT + mutant. (D) Myofibers were electroporated with annexin A6-GFP or mutant A6E233AGFP. Annexin A6E233A cap area (small arrow) was significantly smaller compared with annexin A6 (large arrowhead), correlating with increased FM 4-64 fluorescence area (large arrowhead). Scale bars: $5 \mu \mathrm{m}$. Data are expressed as mean \pm SEM. Differences were assessed by 2-tailed $t$ test (A, C, and D). ${ }^{*} P<0.05$ ( $n=4-18$ myofibers from $n=3$ mice per condition).

membrane lesion (Figure 2A, red channel). These blebs appeared after the formation of repair caps and were seen at the extracellular tip of the repair cap, coincident with FM 4-64 fluorescence (Figure 2, A and B). FM 4-64 is a membrane-impermeant dye that is nonfluorescent in aqueous solution and increases fluorescence intensity as it binds membrane phospholipids exposed during injury; FM 4-64 is commonly used as a marker of membrane injury $(8,13,38,41,42)$. Overexpression of annexin A6 and annexin A2 induced significantly more blebs than were observed after annexin A1 overexpression or GCaMP5G alone (Figure 2, $\mathrm{C}$ and D). Furthermore, annexin-induced blebs were enriched for GCaMP5G, and annexin A6 induced the formation of significantly larger GCaMP5G-containing blebs as compared with annexin A1, A2, or GCaMP5G alone (Figure 2A, green channel, and 2D). A $Z$-stack compilation demonstrated large annexin A6-induced GCaMP5G-positive blebs emanating from the site of injury (Supplemental Video 1). In contrast, annexin A2 resulted in smaller blebs extruding from the repair cap (Supplemental Video 2). These data indicate that annexins not only form a repair cap at the site of membrane disruption, but that these caps serve as sites for excretion of extracellular components enriched for $\mathrm{Ca}^{2+}$-binding proteins.

Decreased intracellular $\mathrm{Ca}^{2+}$ fluorescence at the site of injury with annexin overexpression. Time-lapse imaging of the $\mathrm{Ca}^{2+}$ indicator GCaMP5G after laser injury indicated that intracellular $\mathrm{Ca}^{2+}$ was decreasing concomitantly with extracellular bleb formation, suggesting that these blebs serve to reduce intracellular $\mathrm{Ca}^{2+}$ accumulation through excretion (Supplemental Videos 1 and 2). The annexin-induced reduction in intracellular $\mathrm{Ca}^{2+}$ fluorescence could be seen for all 3 annexins, A1, A2, and A6, but was most evident for annexin A2 and A6 (Figure 3A). Over the 240 seconds of imaging, overexpression of annexin A6 induced the most significant reduction in intracellular $\mathrm{Ca}^{2+}$, visualized as internal GCaMP5G-Ca ${ }^{2+}$ fluorescence (Figure 3B). Detailed analysis of the first 20 seconds after injury showed a significant reduction in internal GCaMP5G$\mathrm{Ca}^{2+}$ fluorescence with annexin A2 and A6, but not annexin A1, when compared with GCaMP5G alone (Figure 3C). Baseline GCaMP5G fluorescence intensity prior to injury was not significantly different between groups (Figure 3D). Reduction in internal $\mathrm{Ca}^{2+}$ fluorescence at the lesion with annexin A6 expression was confirmed using Fluo-4 AM (Supplemental Figure 6). Thus, annex- 
A Expression of annexin A6 from plasmid
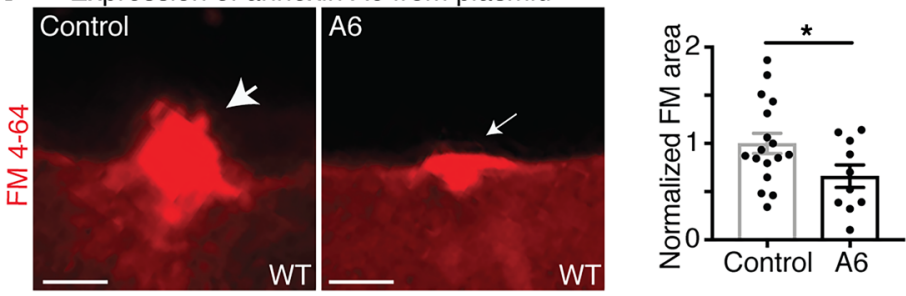

B
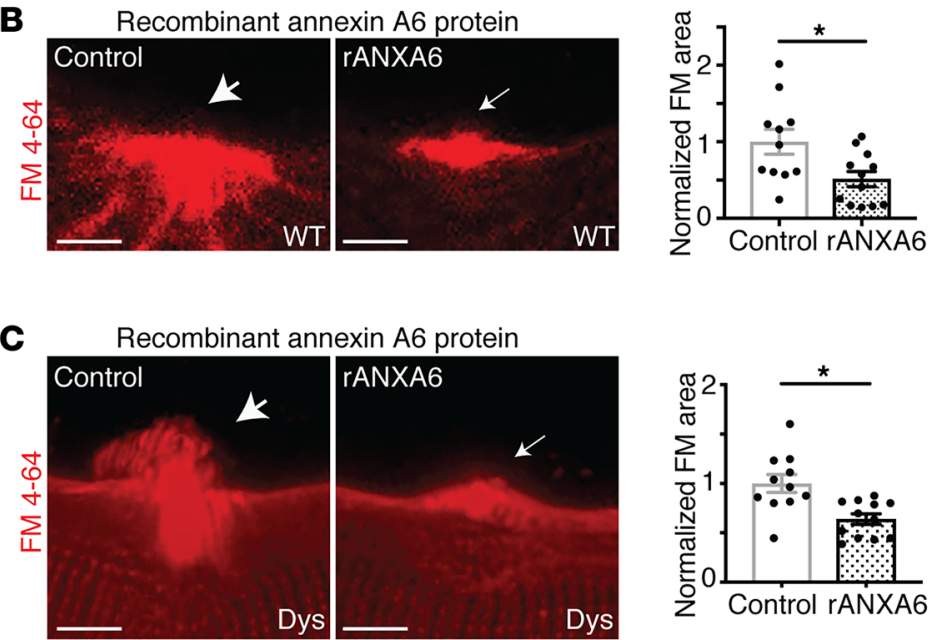

C Recombinant annexin A6 protein

Figure 5. Annexin A6 enhanced membrane repair capacity of healthy and dystrophic myofibers in vitro. (A) Plasmid expression of annexin A6 in wild-type (WT) myofibers reduced FM 4-64 dye uptake, a marker of membrane damage, after laser-induced injury as compared with control myofibers. (B) Wild-type myofibers injured in the presence of extracellular recombinant annexin A6 (rANXA6) had significantly less FM 4-64 dye uptake compared with control myofibers. (C) Dystrophic (Dys) myofibers injured in the presence of rANXA6 had significantly less FM 4-64 dye uptake than control myofibers. Scale bars: $5 \mu \mathrm{m}$. Data are expressed as mean \pm SEM. Differences were assessed by 2 -tailed $t$ test. ${ }^{*} P<0.05(n=10$ myofibers from $n=3$ mice per condition).

generated E233A in annexin A6, to create a similar change in the $\mathrm{Ca}^{2+}$-binding site in the second annexin-repeat domain of annexin A6. Each construct also contained turboGFP or tdTomato at the C-terminus. To assess the effect of homotypic annexin interactions during repair cap formation, myofibers were coelectroporated with wild-type plus wild-type (A6+A6) or wild-type plus mutant (A6+A6E233A) annexin combinations. Mutation of E233 in annexin A6 acted in a dominant-negative fashion, significantly decreasing cap size of the coexpressed wild-type annexin A6 protein (Figure 4A). Prior structural studies suggested that D149 in the first annexin-repeat domain of annexin A6 did not bind $\mathrm{Ca}^{2+}(44)$, and consistent with this, the D149A mutant in annexin A6 had little effect on cap size (Supplemental Figure 8B). The repair cap Feret diameter was plotted as a function of $\mathrm{Ca}^{2+}$ conin expression induced a reduction in $\mathrm{Ca}^{2+}$ signal within the injured myofiber concomitant with enhanced egress of $\mathrm{Ca}^{2+}$-binding-protein-filled blebs. Moreover, annexin A6 was the most effective of the 3 annexins tested at sustaining this response.

Overexpression of $\mathrm{Ca}^{2+}$-binding proteins like annexins may have unexpected effects on intracellular $\mathrm{Ca}^{2+}$ signaling and cellular function. Therefore, we evaluated the $\mathrm{Ca}^{2+}$-handling and contractile properties of isolated myofibers overexpressing annexin A6 compared to controls. Isolated myofibers expressing annexin A6 were loaded with the ratiometric $\mathrm{Ca}^{2+}$ indicator dye Indo-1, and we observed no differences in $\mathrm{Ca}^{2+}$ cycling at 40 or $80 \mathrm{~Hz}$ stimulation frequencies between annexin A6 or control fibers (Supplemental Figure 7, A-C). Unloaded cell shortening was also unaffected by the presence of overexpressed annexin A6 (Supplemental Figure 7, $\mathrm{D}-\mathrm{F})$. These results demonstrate that annexin A6 overexpression was well tolerated by myofibers.

Annexin $\mathrm{A} 6 \mathrm{Ca}^{2+}$ binding is required for repair cap formation and myofiber repair. Mutation of annexin A1 residue D171 and annexin A2 residue D161 was previously shown to inhibit annexin membrane translocation in HEK cells $(28,43)$. We queried whether these mutations would inhibit translocation and formation of the macromolecular annexin repair cap formed after muscle membrane injury in live myofibers. Alignment of annexins A1, A2, and A6 protein sequences was used to identify the conserved residues within the consensus sequence of type II $\mathrm{Ca}^{2+}$-binding sites across all 3 annexin proteins (Supplemental Figure 2). In order to disrupt $\mathrm{Ca}^{2+}$ binding in annexin A1, A2, and A6, site-directed mutagenesis was performed to convert the aspartic acid residue in the first type II $\mathrm{Ca}^{2+}$-binding site into an alanine residue (A1D171A, A2D161A, and A6D149A, respectively) (Supplemental Figure 8A). We also centration using a modified Hill equation. Expression of mutant annexin A6E233A significantly reduced the cap diameter $\left(D_{M A X}\right)$ of the coexpressed wild-type annexin A6 protein (Figure 4B). To assess the effect of heterotypic annexin interactions on repair cap formation, myofibers were coelectroporated with various combinations of wild-type and mutant annexin constructs. Coexpression of mutant annexin A6E233A resulted in a significant reduction in annexin A1, A2, and A6 cap size compared with A1+A6, $\mathrm{A} 2+\mathrm{A} 6$, and A6+A6 controls, respectively (Figure 4C). Together, these data show that annexin proteins interact in a homotypic and heterotypic fashion, influencing annexin repair-complex assembly and that the mutant annexin A6 protein is sufficient to negatively modulate annexin-complex assembly during repair.

$\mathrm{Ca}^{2+}$ binding of both annexin $\mathrm{A} 1$ and $\mathrm{A} 2$ was also required for repair cap formation. A1D171A and A2D161A mutant cap size was reduced compared with wild-type annexin A1 and A2 controls, respectively. Expression of mutant annexin A1D171A and A2D161A significantly reduced the repair cap diameter $\left(\mathrm{D}_{\mathrm{MAX}}\right)$ of the respective coexpressed wild-type annexin protein (Supplemental Figure 8B). Despite the ability of mutant annexin A1D171A and A2D161A to significantly decrease coexpressed wild-type annexin A1 and A2 cap size, respectively, A1D171A or A2D171A had minimal effect on wild-type annexin A6 cap size (Supplemental Figure $8 \mathrm{C}$ ). These data show that annexin A1 and A2 interact in a homotypic fashion to influence self-cap assembly, while A6 localization to the repair cap is minimally modulated by annexin A1 and A2 localization.

To determine the effect of dominant-negative annexin A6 on the assembly of annexins A1, A2, and A6 at the repair cap and membrane repair capacity, laser injury was similarly performed on isolated myofibers in the presence of FM 4-64. Myofibers expressing annex- 

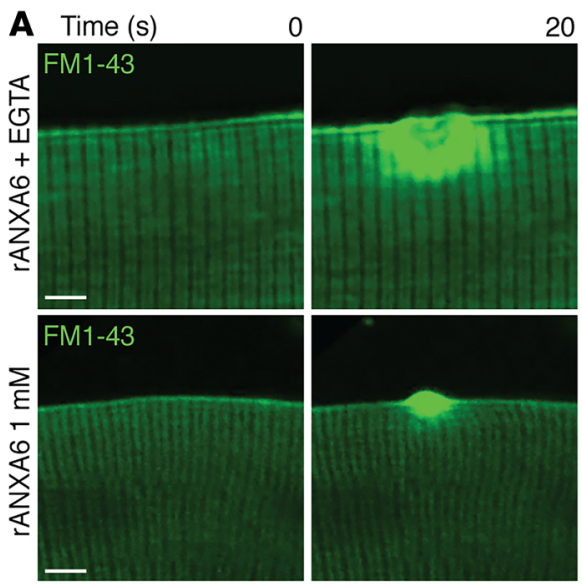

20
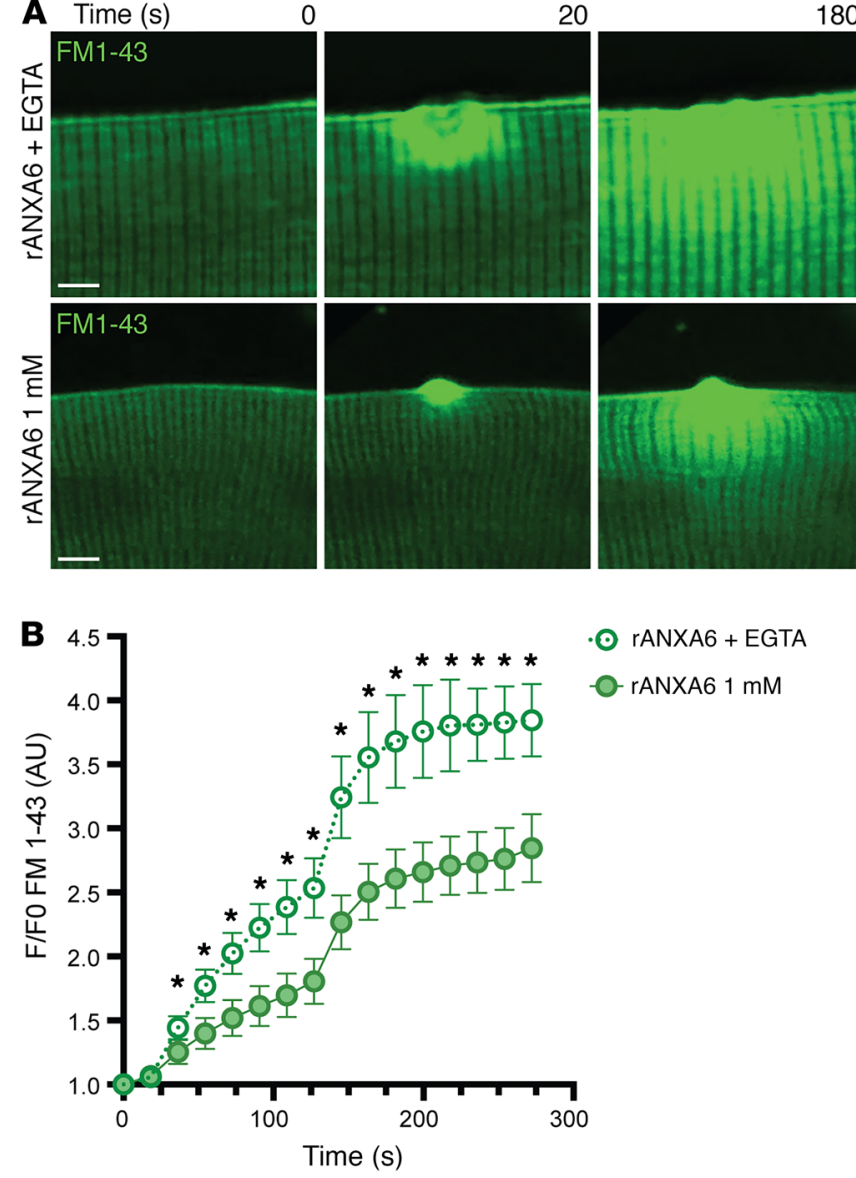

in A6E233A-GFP had increased FM 4-64 fluorescence area after laser injury compared with control myofibers expressing wild-type annexin A6-GFP (Figure 4D). These results indicate that a functional annexin repair complex is required for proper membrane repair and annexin A6 participates in orchestrating complex formation.

Annexin A6 protected against laser-induced myofiber injury in vitro in a $\mathrm{Ca}^{2+}$-dependent manner. Since annexin A6 facilitates the formation of the macromolecular repair cap complex and was the most efficient at forming large, $\mathrm{Ca}^{2+}$-filled blebs at the site of membrane injury, we assessed whether expression of annexin A6 would reduce membrane injury in wild-type myofibers. Wild-type myofibers were electroporated with annexin A6-GFP or mock electroporated and then laser damaged in the presence of FM 4-64 to mark the injury area. Wild-type myofibers overexpressing annexin A6 had decreased FM 4-64 dye uptake after laser-induced membrane injury compared with control myofibers (Figure 5A). These results indicate that intracellular overexpression of annexin A6 is effective at improving membrane repair and/or protecting against laser-induced membrane injury in isolated myofibers.

Since intracellular annexin A6 targets phospholipids exposed at the site of membrane injury and enhances membrane repair capacity, we hypothesized that extracellular recombinant annexin A6 would also localize to the site of injury and protect against membrane injury. Muscular dystrophy is a progressive muscle-wasting disease, arising from loss-of-function mutations in critical cytoskeletal or membrane-associated proteins, often resulting in fragile plasma membranes. To determine if recombinant annexin A6
Figure 6. $\mathrm{Ca}^{2+}$ dependency of the protective effects of recombinant annexin A6. (A and B) Wild-type myofibers were isolated and loaded with a fluorescence marker of membrane damage, FM 1-43 (green). Myofibers were pretreated with recombinant annexin $A 6$ ( $r A N X A 6)$ and then damaged in $1 \mathrm{mM} \mathrm{Ca}^{2+}$ solution or $0 \mathrm{mM} \mathrm{Ca}^{2+}$ plus EGTA, a calcium chelator. FM 1-43 fluorescence uptake over time was significantly reduced at $1 \mathrm{mM}$ $\mathrm{Ca}^{2+}$ compared with when EGTA was present. Scale bars: $5 \mu \mathrm{m}$. Data are expressed as mean \pm SEM. Differences were tested by 2-way ANOVA with Bonferroni's multiple-comparisons test. ${ }^{*} P<0.05(n=10$ myofibers per condition; $n=3$ mice per condition).

could protect against membrane insult in both healthy and dystrophic muscle, wild-type and dystrophic myofibers from a model representing Duchenne muscular dystrophy were isolated and incubated with recombinant annexin A6 or vehicle control. Laser injury was conducted in the presence of FM 4-64 to visualize the extent of injury. Treatment with extracellular recombinant annexin A6 reduced FM 4-64 fluorescence area compared with vehicle control-treated myofibers, indicating enhanced repair in both healthy and dystrophic myofibers (Figure 5, B and C).

To assess whether recombinant annexin A6's protective effects required external $\mathrm{Ca}^{2+}$, wild-type myofibers were pretreated with recombinant annexin A6, loaded with FM 1-43, a fluorescent marker of membrane damage similar to FM 4-64, and subsequently damaged in solution containing $1 \mathrm{mM} \mathrm{Ca}^{2+}$ or $0 \mathrm{mM} \mathrm{Ca}^{2+}$ plus EGTA, a $\mathrm{Ca}^{2+}$ chelator. FM 1-43 fluorescence accumulation at the lesion over time (F/FO) was significantly increased in the absence of $\mathrm{Ca}^{2+}$ compared with that in the presence of $1 \mathrm{mM} \mathrm{Ca}^{2+}$ (Figure 6, $\mathrm{A}$ and $\mathrm{B}$ ). These data demonstrate that extracellular recombinant annexin A6 protects against membrane injury and/or enhances repair through extracellular exposure in a $\mathrm{Ca}^{2+}$-dependent manner.

Recombinant annexin A6 protected against acute muscle injury in vivo. To determine the therapeutic potential of recombinant annexin A6 to protect against muscle injury in vivo, recombinant annexin A6 was utilized as a tool compound. Recombinant annexin A6 or vehicle control was injected intramuscularly into the tibialis anterior muscles of wild-type mice 2 hours prior to toxin-induced injury. Mice were injected intraperitoneally with Evans blue dye, a vital tracer that is excluded by intact healthy myofibers but is readily taken up in injured, permeable myofibers (45). Three hours after cardiotoxin injury muscle was evaluated for Evans blue dye uptake (Figure 7A). Gross imaging showed that pretreatment with recombinant annexin A6 reduced cardiotoxin-induced muscle damage in vivo, as seen as less dye (blue) uptake compared with controls (Figure 7B). Fluorescence imaging showed a 50\% decrease in dye (red) uptake with recombinant annexin A6 pretreatment compared with control muscle (Figure 7C). Surface plot profiles illustrate reduced dye fluorescence in tibialis anterior muscle pretreated with intramuscular recombinant annexin A6 (Figure 7D).

Although intramuscular injection of annexin A6 was effective at reducing acute injury, this route of application is not optimal for large muscle groups, internal tissues, or treatment of chronic diseases. Therefore, we examined the efficacy of recombinant annexin A6 administered systemically via retro-orbital injection in the protection against acute muscle injury. Recombinant annexin A6 or vehicle was injected into the retro-orbital cavity 2 hours prior to toxin-induced injury. Mice were simultaneously injected 
A Pretreatment injection (i.m.)

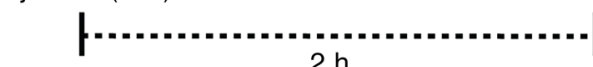

Induce injury

Inject dye (i.p.)

$2 \mathrm{~h}$<smiles>[3H][3H]</smiles><smiles>[3H][IH]</smiles>

$3 \mathrm{~h}$

Harvest

B
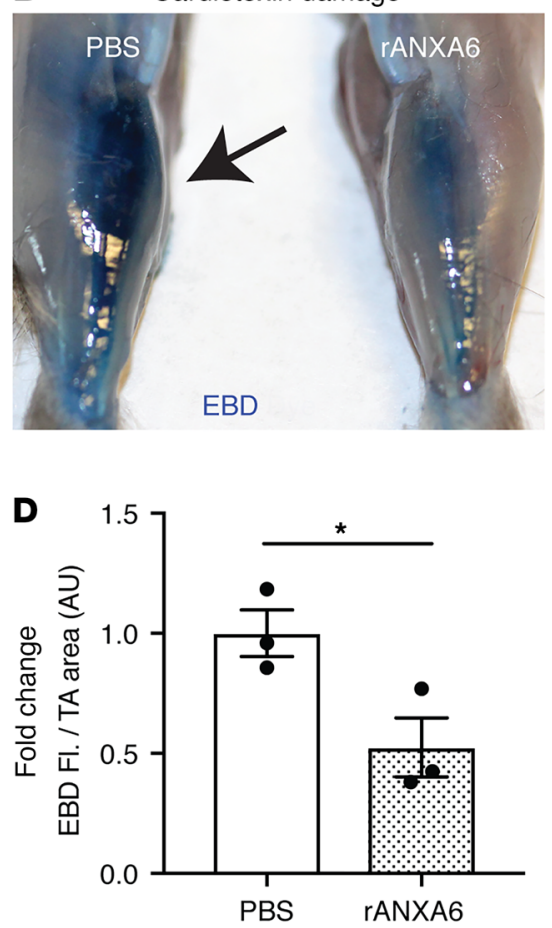

C

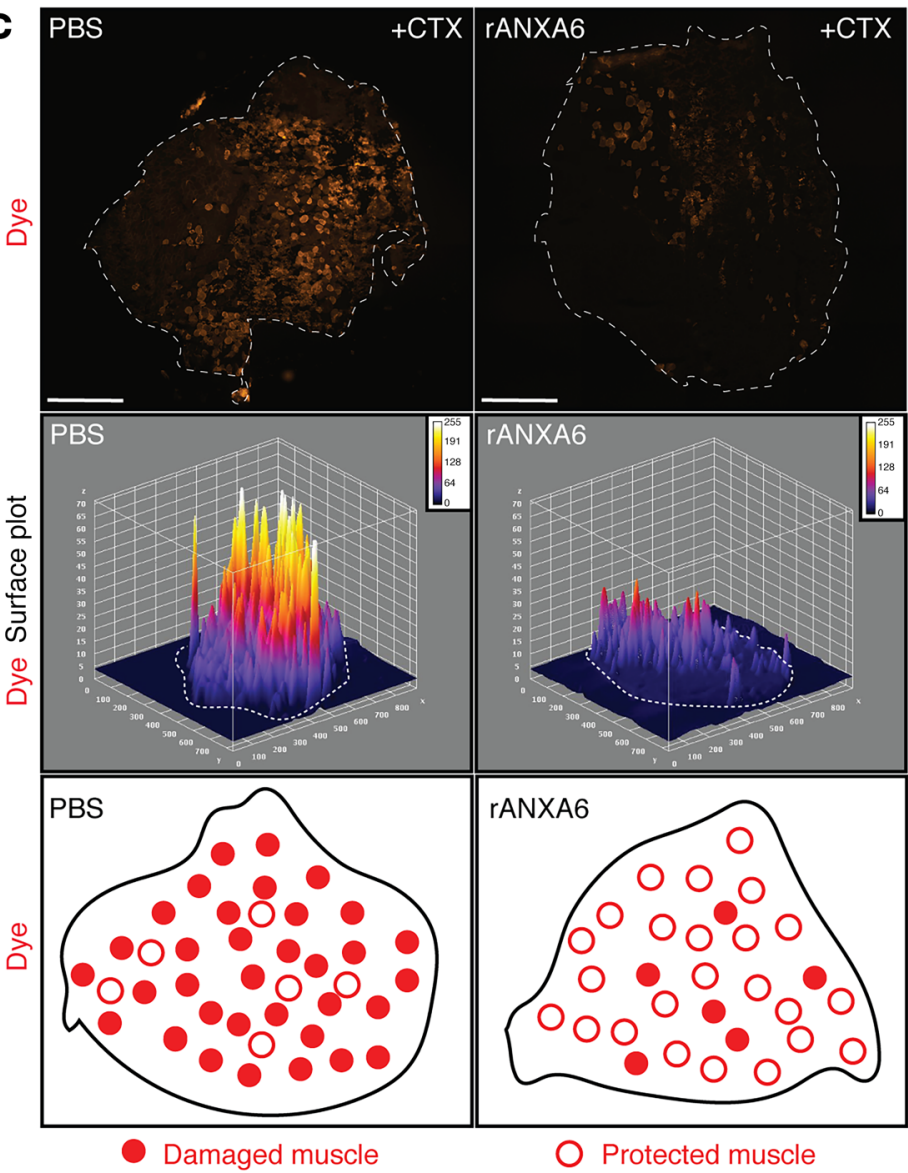

Figure 7. Local delivery using intramuscular injection of recombinant annexin A6 protected against muscle damage in vivo. (A) Tibialis anterior muscles of wild-type mice were injected i.m. with recombinant human annexin A6 (rANXA6) or vehicle control and subsequently injured with cardiotoxin injection. (B) Gross imaging revealed decreased Evans blue dye (blue) uptake in rANXA6-pretreated muscle compared with the contralateral control muscle. (C) Immunofluorescence imaging revealed decreased dye uptake (red) in muscle pretreated with rANXA6. Surface plots of dye uptake depict reduced fluorescence in muscle pretreated with rANXA6. White dotted lines outline the muscle sections. (D) Tibialis anterior (TA) muscle pretreated with rANXA6 had a significant reduction, approximately 50\%, of Evans blue dye fluorescence over muscle area compared with control muscle. Scale bars: 1 mm. Data are expressed as mean \pm SEM. Differences were assessed by 2 -tailed $t$ test. ${ }^{*} P<0.05$ ( $n=3$ mice per condition). EBD, Evans blue dye.

with Evans blue dye. Three hours after cardiotoxin injury muscle was evaluated for Evans blue dye uptake (Figure 8A). Fluorescence imaging showed a $38 \%$ decrease in dye (red) uptake with recombinant annexin A6 pretreatment compared with vehicle control (Figure 8, B and C). Surface plot profiles illustrate reduced dye fluorescence in muscle pretreated with systemic recombinant annexin A6 (Figure 8C). In addition, whole-tissue spectroscopic analysis of injured gastrocnemius/soleus muscles revealed a $58 \%$ reduction in dye uptake with extracellular recombinant annexin A6 pretreatment compared with vehicle treated (Figure 8D). These results demonstrate that local and systemic administration of recombinant annexin A6 protects against acute muscle injury in vivo.

To determine if recombinant annexin A6 administration enhanced myofiber survival and/or recovery from injury, mice were administered recombinant annexin A6 or BSA control, at 1 $\mathrm{mg} / \mathrm{kg}$, through retro-orbital systemic injection. Two hours after protein administration, cardiotoxin was injected into the tibialis anterior muscles to induce focal muscle injury. Muscle was harvested 7 days after injury and histology evaluated for injury area (Figure 8E). Pretreatment with recombinant annexin A6 reduced the percentage of injured muscle, marked by internal myonuclei (black dotted outline), at 7 days after insult (Figure 8, F and G). These data illustrate that systemic administration of recombinant annexin A6 protects against acute muscle injury, enhancing myocyte survival and/or recovery from injury.

Recombinant annexin A6 protected against chronic muscle inju$r y$ in vivo. We next assessed the ability of recombinant annexin A6, administered systemically, to protect against muscle damage in the $\mathrm{S} g c g$-null mouse model of limb girdle muscular dystrophy type 2C (LGMD2C) (46). Sgcg-null mice lack $\gamma$-sarcoglycan, an 
A Pretreatment Induce injury

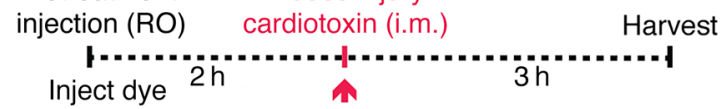

B
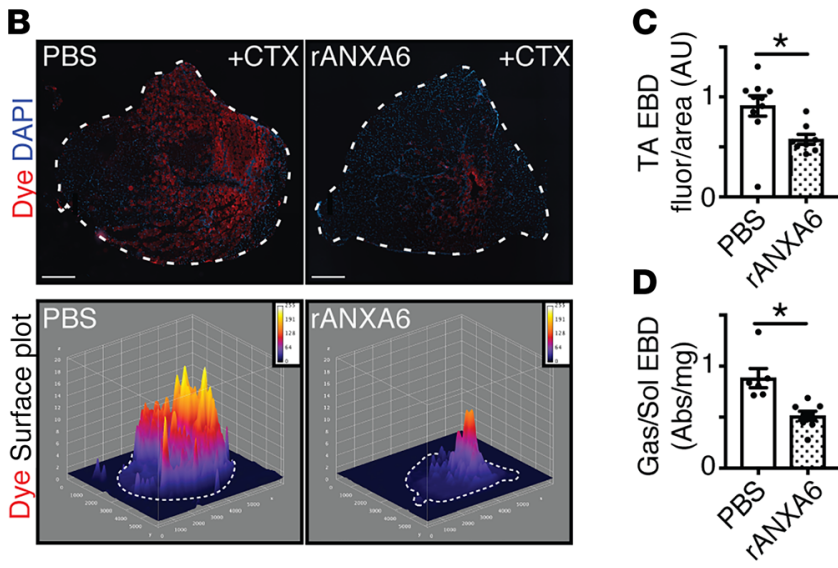

$\mathbf{E}$
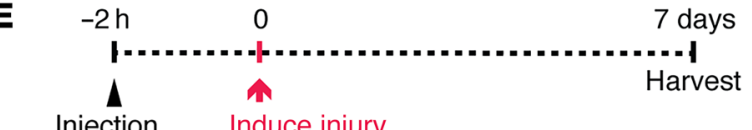

Injection Induce injury

$1 \mathrm{mg} / \mathrm{kg}(\mathrm{RO})$ cardiotoxin (i.m.)

$\mathbf{F}$
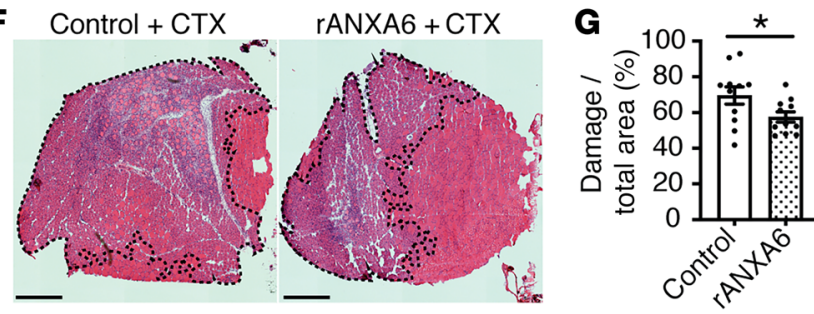

integral membrane component of the dystrophin glycoprotein complex required for membrane stability and function. Humans and mice lacking $\gamma$-sarcoglycan develop progressive muscle disease, reduced muscle function, and elevated serum creatine kinase (CK), a serum biomarker of muscle injury and membrane leak. To determine if recombinant annexin A6 protected in dystrophic muscle, $S g c g$-null mice were treated systemically with recombinant annexin A6 or BSA control over 48 hours (Figure 9A). Mice were then subjected to 60 minutes of treadmill running to induce physiological muscle damage and CK release. Recombinant annexin A6 reduced the fold change of CK after exercise normalized to pretreatment levels, consistent with improved membrane resealing (Figure 9, A and B). We also injected recombinant annexin A6 over a 2-week interval in Sgcg-null mice. Recombinant annexin A6 was injected (1 $\mathrm{mg} / \mathrm{kg}$ ) once every 3 days for 14 days (Figure 9C). Administration of recombinant annexin A6 significantly decreased levels of serum CK compared with control treatment at day 14 (Figure 9D). Annexin A6 was more effective than annexin A2 at reducing intracellular $\mathrm{Ca}^{2+}$ after injury (Figure 2D). Using the same 14-day dosing regimen in $S g c g$-null mice, short-term systemic administration of recombinant annexin A6 significantly reduced serum CK levels compared with recombinant annexin A2 (Figure 9E). The gastrocnemius/soleus muscle from recombinant annexin-
Figure 8. Systemic delivery using retro-orbital injection of recombinant annexin A6 protected against muscle damage in vivo. (A) Wildtype mice were injected retro-orbitally $(\mathrm{RO})$ with recombinant human annexin A6 ( $r A N X A 6)$ or control solution. Following this, muscles were damaged with cardiotoxin (CTX). (B and C) Immunofluorescence imaging revealed approximately $38 \%$ less dye uptake (red) in muscle pretreated with rANXA6. Dotted lines outline the tibialis anterior muscle sections (top panel). DAPI (blue) marks nuclei. Surface plots of dye uptake depict reduced fluorescence in muscle pretreated with rANXA6. (D) Whole-tissue spectroscopic analysis of injured gastrocnemius/soleus muscles revealed a $58 \%$ reduction in dye uptake with rANXA6 pretreatment compared with control muscle. Abs, absorbance at $620 \mathrm{~nm}$. (E) Wild-type mice were injected intravenously with rANXA6 or control solution. Two hours later, tibialis anterior muscles were damaged with cardiotoxin. Muscles were harvested 7 days after injury. (F and $\mathbf{G}$ ) Hematoxylin and eosin images were quantified and show a reduction in percentage myofiber damage (dotted lines), in rANXA6-treated mice compared with controls. Scale bars: $1 \mathrm{~mm}$ (B) and $500 \mu \mathrm{m}$ (F). Data are expressed as mean \pm SEM. Differences were assessed by 2-tailed $t$ test. ${ }^{*} P<0.05 . n=3$ mice, $n=6$ legs per condition (B-D); $n=6$ mice; $n=11$ muscles per condition (F and $\mathbf{C}$ ). EBD, Evans blue dye; TA, tibialis anterior.

treated mice showed qualitatively less injury (Figure 9F). These data indicate that extracellular recombinant annexin A6 protects against injury or enhances repair in chronically injured, dystrophic mouse muscle, in vivo.

\section{Discussion}

Annexins promote calcium-filled bleb formation at the site of membrane injury. Plasma membrane instability is inherent to many forms of muscular dystrophy and thought to contribute to dysregulated $\mathrm{Ca}^{2+}$ homoeostasis and disease pathogenesis. Molkentin and colleagues showed that transgenic overexpression of transient receptor potential cation channel subfamily $\mathrm{C}$ member 3 (TRPC3) was sufficient to increase myofiber $\mathrm{Ca}^{2+}$ influx and result in a dystrophy-like phenotype (47). Correspondingly, transgenic overexpression of sarcoplasmic/endoplasmic reticulum $\mathrm{Ca}^{2+}$-ATPase (SERCA1) reduced cytosolic $\mathrm{Ca}^{2+}$ levels and mitigated dystrophic muscle pathology, implicating $\mathrm{Ca}^{2+}$ in disease progression (48). We found that increased expression of annexins in muscle fibers decreased injury-associated $\mathrm{Ca}^{2+}$ fluorescence within myofibers. This reduction of $\mathrm{Ca}^{2+}$-associated fluorescence was at the injury site and correlated with extracellular bleb formation emanating from annexin repair caps. Both annexin A2 and A6 could induce the formation of membranous blebs containing the $\mathrm{Ca}^{2+}$-binding protein GCaMP5G. Furthermore, overexpression of annexins A1, A2, and A6 each reduced endpoint $\mathrm{Ca}^{2+}$ fluorescence accumulation within the myofiber after injury. Of the 3 annexins tested, annexin A6 overexpression resulted in the most sustained effect on reducing the formation of large GCaMP5G-containing blebs induced by injury-associated $\mathrm{Ca}^{2+}$ accumulation. In HEK293 cells damaged with streptolysin O (SLO), the presence of extracellular membranous blebs correlated with increased cell survival and reduction in cytoplasmic $\mathrm{Ca}^{2+}$ levels, a process facilitated by annexin A1 (7). Davenport and colleagues showed that overexpression of annexin A1-GFP in injured Xenopus oocytes resulted in annexin A1-positive blebs originating from the site of damage (30). However, the effects of annexin A2 or A6 overexpression were not 
A

Acute dosing: Sgcg-null

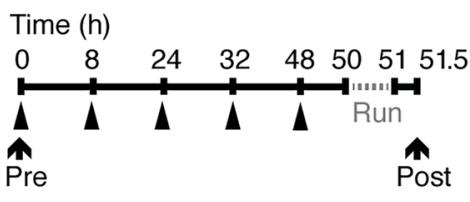

$\Delta$ Injection $1 \mathrm{mg} / \mathrm{kg}$

C

14-day dosing

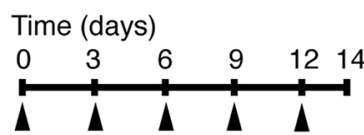

$\Delta$ injection $1 \mathrm{mg} / \mathrm{kg}$ or PBS

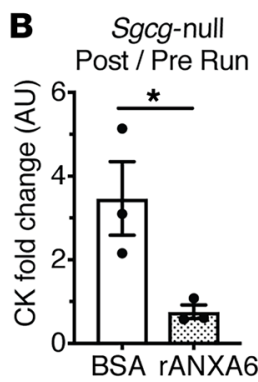

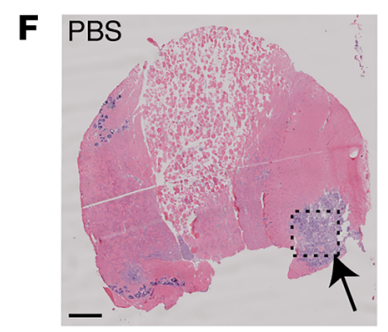
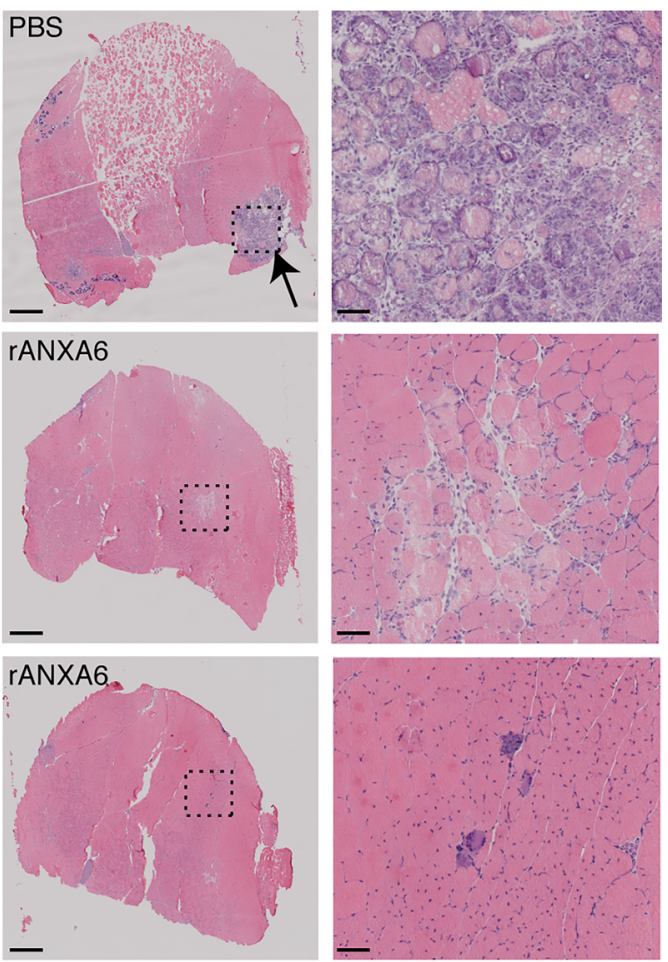

Figure 9. Recombinant annexin A6 protected against muscle damage in a mouse model of muscular dystrophy in vivo. (A and B) Sgcg-null mice, a model of limb girdle muscular dystrophy 2C, were injected intravenously with recombinant human annexin A6 (rANXA6) or BSA control solution 5 times over 48 hours. Prior to injections, serum creatine kinase (CK) was measured. Two hours after the fifth injection, mice were subjected to 60 minutes of downhill running. Thirty minutes after exercise, serum CK was measured. The fold change in CK after/before running was significantly reduced with rANXA6 administration compared with BSA-injected controls, consistent with a reduction in muscle injury from acute running. (C and D) Sgcg-null mice were injected intravenously every 3 days over 14 days with rANXA6 or control. On day 14, serum CK was evaluated. Serum CK in Sgcg-null mice treated with rANXA6 was lower than PBS control. (E) Sgcg-null mice were injected intravenously every 3 days for 14 days with rANXA6 or recombinant annexin A2. The serum CK fold change after/before treatment (day 14/day 0) was significantly reduced in Sgcg-null mice treated with recombinant annexin A6 compared with annexin A2. (F) Histological analysis of gastrocnemius/soleus muscles from Sgcg-null mice shown in part $\mathbf{D}$ injected with PBS or recombinant annexin A6. Low magnification is on the left and high magnification of boxed areas is on the right. Scale bars: $500 \mu \mathrm{m}$ (left) and $50 \mu \mathrm{m}$ (right). Data are expressed as mean \pm SEM. Differences were assessed by 2-tailed $t$ test (B and $\mathbf{E}) .{ }^{*} P<0.05$ ( $n=3$ mice per condition, except part $\mathbf{D}$, in which $n=2 \mathrm{WT}$ controls, 1 Sgcg-null control, and 2 Sgcg-null treated mice).

assessed in either study. These data combined suggest that bleb formation as a mechanism of membrane repair is conserved across species and tissue types and is facilitated by the presence of annexin proteins.

We envision a model in which annexin A6 facilitates cytoplasmic $\mathrm{Ca}^{2+}$ and protein excretion into extracellular blebs whose formation is further induced by annexin A1 and annexin A2. In artificial membrane patches, the presence of annexin A1 or annexin A2 induced bleb formation at sites of membrane imperfection (18). In contrast, the presence of annexin A6 induced $\mathrm{Ca}^{2+}$-dependent contraction of an artificial membrane into large folds (18). The difference between annexin A6 inducing blebs in live myofibers or folds in an artificial membrane may reflect the presence of endogenously expressed annexin $\mathrm{A} 1$ and $\mathrm{A} 2$ in isolated myofibers compared with exposure to a single recombinant annexin protein in the artificial membrane studies. We hypothesize that within the macromolecular repair complex, multiple annexins actively participate in bleb formation, which acts to remove large membrane lesions and facilitates wound closure, excision of damaged membrane, and reduction of $\mathrm{Ca}^{2+}$ at the injury site.
Annexin A6 protects against muscle membrane injury and enhances membrane repair. We showed that annexin proteins, including annexin A1, A2, and A6, localize to the site of membrane injury, facilitating membrane repair cap and bleb formation. Mutation of annexin A6 abrogated repair cap formation, decreasing repair capacity, resulting in increased dye uptake. On the other hand, pretreatment with recombinant annexin A6 reduced dye uptake after laser-induced muscle injury and after toxin-induced muscle injury in vivo. Our current data, however, do not distinguish between annexin $\mathrm{A} 6$ enhancing membrane repair, reducing membrane injury, or a combination of both mechanisms. As a therapeutic tool, enhancing the cells' ability to repair and/or reduce injury through stabilizing the cell membrane are both beneficial avenues that can lead to improved cell survival. Our previous studies have shown that annexin A6 is upregulated in muscle from models of chronic muscular dystrophy $(33,35,49)$. Proteomic profiling of $m d x$ mouse muscle showed that annexins $\mathrm{A} 1$ and $\mathrm{A} 2$ are enriched in $m d x$ muscle membrane, consistent with a role for annexins at the membrane of injured muscle cells (50). Annexins bind membrane phospholipids, including phosphatidylserine, which is exposed during membrane disruption. Phosphatidylserine rear- 
rangement after injury provides an optimal binding target for extracellular annexins to facilitate membrane folding, blebbing, and rolling at sites of membrane damage and imperfection (18). We hypothesize that upregulation of annexins is a compensatory mechanism to facilitate excision of defective membrane in fibers undergoing chronic damage.

Additional studies of cardiac muscle injury further suggest a role for annexin proteins in modulating the repair response. Administration of recombinant annexin A1 or the N-terminal annexin A1 peptide (AC2-26) elicited a cardioprotective response in a rat model of myocardial ischemia-reperfusion-induced injury (51). Meng et al. demonstrated that downregulation of annexin A3 resulted in cardioprotection, decreasing rat myocardial infarct size through activation of AKT signaling (52). In addition to membrane reorganization, annexins act as scaffolds regulating multiple downstream intracellular signaling cascades important for orchestrating repair from injury. Both intra- and extracellular functions of annexin proteins should be considered when evaluating the therapeutic potential of annexin proteins.

The studies presented herein indicate that human recombinant annexin A6 protein may be a suitable biologic to protect against acute muscle injury. We showed that human recombinant annexin A6 was capable of resealing injured membrane in mouse models, confirming functional activity of the human recombinant protein in a mouse preclinical model. Human and mouse annexin A6 proteins are $94.35 \%$ identical at the amino acid level, with increasing percentages of amino acid conservation between humans and rat (94.65\%), dog (95.54\%), and monkey (98.96\%), and this high degree of similarity is consistent with the human recombinant protein having efficacy in a mouse model (Supplemental Figure 9). The current studies are limited by recombinant protein available, and further studies are needed to determine if recombinant annexin A6 can facilitate membrane repair and reduce the susceptibility to injury long-term in chronic models of muscle disease and in tissues beyond skeletal muscle. Annexin A6 was originally identified in a mouse model of muscular dystrophy as a genetic modifier of muscle membrane leak, and annexin A6 was subsequently shown to modify injury response in healthy mouse muscle (35). We hypothesize that enhancing repair through administration of recombinant annexin A6 protein will provide similar protection in dystrophic muscle but would require longterm intermittent dosing. Future studies will require optimizing mammalian recombinant annexin A6 protein production to generate sufficient quantities of purified protein.

Combinatorial approaches to improve membrane repair. We now described recombinant annexin A6 and its ability to protect normal and dystrophic muscle from laser-induced membrane injury. In addition, both intramuscular and systemic administration of recombinant annexin A6 protected against toxin-induced muscle membrane injury in vivo. We previously found that glucocorticoid administration increased annexin expression in muscle, and this correlated with enhanced muscle repair in multiple mouse models of muscular dystrophy including $m d x$ (Duchenne), dysferlin-null (LGMD2B), and $\gamma$-sarcoglycan-null (LGMD2C) mice $(36,53)$. Glucocorticoid treatment also increased the expression of the Trim72 gene that encodes mitsugumin 53 (known as MG53), a repair protein that localizes to the site of membrane injury and considered a "molecular band-aid" for improving cellular wound healing. Similar to the annexins, MG53 is upregulated in chronic muscle injury and enhances repair in dystrophic muscles, as well as other tissues like heart, lung, and kidney (54-59). MG53 is a component of the annexin-mediated repair complex, localizing adjacent to the annexin repair cap (5). Additional studies are required to determine if coadministration of recombinant annexin A6 with glucocorticoids and/or MG53 will further strengthen the clinical relevance of these putative therapeutics for conditions resulting from membrane lesions.

\section{Methods}

Animals. Wild-type mice from the 129T2/SvEmsJ background were bred and housed in a specific pathogen-free facility on a 12-hour light/12-hour dark cycle and fed ad libitum in accordance with the Northwestern University's Institutional Animal Care and Use Committee regulations. 129T2/SvEmsJ (129T2) mice were purchased from the Jackson Laboratory (stock 002065). $m d x /$ hLTBP 4 mice were generated as described previously $(34,60)$. Sgcg-null mice were generated as described in Hack et al. (46). Two- to 3-monthold males and females were used for all wild-type mouse experiments. Sgcg-null cohorts were age and sex matched with mice between 2 and 5 months old.

Plasmids. Plasmids encoding annexin A1, A2, and A6 with a carboxyl-terminal turboGFP tag were obtained from Origene. Subcloning of annexin A1, A2, and A6 to replace the GFP tag with tdTomato (Addgene) was performed by Mutagenix. Site-directed mutagenesis was performed by Mutagenix on annexin A1-GFP, A2-GFP, and A6-GFP to create the $\mathrm{Ca}^{2+}$-binding mutants A1-D171A-GFP, A2-D161A-GFP, A6-D149A-GFP, and A6-E233A-GFP. Constructs were sequenced to verify mutagenesis. Plasmid DNA was isolated using the EndoFree Plasmid Maxi Kit (Qiagen, 12362). The $\mathrm{Ca}^{2+}$ sensor GCaMP5G was purchased from Addgene (catalog 31788).

Sequence comparison and protein schematics. Protein ribbon diagrams were generated using Swiss-PdbViewer using solved crystal structures of annexin A1 (1MCX), annexin A2 (2HYW), and annexin A6 (1AVC) available on www.rcsb.org. Clustal Omega from the European Bioinformatics Institute (EMBL-EBI) was used to align the sequences (from www.ncbi.nlm.nih.gov) annexin A1 (NM_010730), annexin A2 (NM_007585), annexin A6 (NM_013472), and annexin A6-encoding sequencing from multiple species (Homo sapiens [AAH17046.1], Macaca mulatta [AFE65315.1], Canis lupus [XP_00519331.1], Rattus norvegicus [NP_07707.2], and Mus musculus [NP_038500.2]).

Electroporation, myofiber isolation, laser injury, and cap and vesicle measurement. Flexor digitorum brevis (FDB) fibers were transfected with endotoxin-free plasmid DNA by in vivo electroporation. The utilized methods were described previously $(5,38,61)$. $Z$-stack projections were acquired from consecutive acquisitions after the final timelapse frame, approximately 4 minutes after damage, with a $0.125-\mu \mathrm{m}$ step size between slices. $Z$-stack renderings were constructed in FIJI (NIH). Measurement of the cap area and Feret diameter was conducted from a single slice near the middle of the $Z$-stack using FIJI imaging tools. Fibers expressing similar levels of tagged or GCaMP5G protein were compared. GCaMP5G-Ca ${ }^{2+}$ fluorescence was measured from the acquired time-lapse images, using a standard rectangular region of interest, placed inside the myofiber below the site of damage using FIJI. Fluorescence is expressed as F/FO. External vesicle number and 
GCaMP5G area were measured from endpoint $Z$-stacks and max-projection images using FIJI. Vesicles were considered external if they were found outside the sarcolemma assessed in brightfield and fluorescence channels. All measurements were acquired from myofibers isolated from at least 3 mice, $n=3$ myofibers per mouse.

For recombinant myofiber studies, myofibers were isolated from $m d x /$ hLTBP4 mice as described above. Myofibers were incubated in Ringer's media with or without $25 \mu \mathrm{g} / \mathrm{mL}$ recombinant annexin A6 (5186-A6-050, R\&D Systems). FM 4-64 (2.5 $\mu \mathrm{M})$ was added to the myofibers just prior to imaging. Images were acquired and quantitated as described above. The FM 4-64 area was measured using FIJI at imaging endpoint from a single slice near the middle of the $Z$-stack. $Z$-stack step size $(0.125 \mu \mathrm{m})$ was acquired from cap end to end.

Myofiber quality control was based on a number of characteristics, including using adherent myofibers with intact sarcomere structure detected through brightfield imaging. Myofibers appeared devoid of tears or ruptures induced during the isolation protocol. The region of the myofiber selected for damage was linear and not located on a nucleus or neuromuscular junction. Additionally, fluorescence intensity within both the red and green channels suggested similar expression levels prior to damage.

Multiphoton laser injury and imaging. Fibers were subjected to laser-induced damage at room temperature using the Nikon A1R$\mathrm{MP}+$ multiphoton microscope. Imaging was performed using a $\times 25$ 1.1-NA objective directed by the NIS-Elements AR imaging software. GFP and FM 4-64 were excited using a 920-nm-wavelength laser and emission wavelengths of $575 \mathrm{~nm}$ and $629 \mathrm{~nm}$ were collected, respectively. To induce laser damage on isolated myofibers, a diffraction-limited spot (diameter approximately $410 \mathrm{~nm}$ ) was created on the lateral membrane of the myofiber using a 920-nm-wavelength laser at $10 \%-15 \%$ laser power for 1 second. Time-lapse images were collected as follows: one image was collected prior to damage, one image upon damage, and then every 8 seconds for 80 seconds (10 images) followed by every 30 seconds for 5 minutes (10 images). At the end of the time-lapsed image series, $Z$-stack images were collected at 250$\mathrm{nm}$ intervals through the damaged site on the myofiber directed by the NIS-Elements AR imaging software. The multiphoton microscope was used to acquire data presented in Supplemental Figure 5, Figure $4 \mathrm{D}$, and Figure 6.

For recombinant protein and calcium studies, myofibers were isolated from wild-type mice as described above. Myofibers were incubated in $20 \mu \mathrm{g} / \mathrm{mL}$ recombinant annexin A6 (5186-A6-050, R\&D Systems) in $1 \mathrm{mM} \mathrm{Ca}^{2+}$ Ringer's or $0 \mathrm{mM} \mathrm{Ca}^{2+}$ plus EGTA. FM 1-43 (2.5 $\mu \mathrm{M})$ was added to the myofibers just prior to imaging. Images were acquired and quantitated as described above. FM 1-43 fluorescence over time was measured using FIJI and plotted over time as F/FO.

Cardiotoxin injury and analysis. Tibialis anterior muscles of wildtype mice were injected with $25 \mu \mathrm{g} / \mathrm{mL}$ recombinant annexin A6 (5186-A6-050, R\&D Systems) or Ringer's in sedated mice (3\% isoflurane, $0.8 \mathrm{~L} / \mathrm{min}_{2}$ ). For systemic administration, wild-type mice were injected with $1 \mathrm{mg} / \mathrm{kg}$ recombinant annexin A6 (5186-A6-050, R\&D Systems) or PBS diluted in Evans blue dye at $5 \mu \mathrm{L} / \mathrm{g}$ body weight (E-2129, Sigma-Aldrich) into the retro-orbital cavity of sedated mice (3\% isoflurane, $0.8 \mathrm{~L} / \mathrm{min}_{2}$ ). Additionally, mice were injected with Evans blue dye at $5 \mu \mathrm{L} / \mathrm{g}$ body weight dissolved in PBS at $10 \mathrm{mg} / \mathrm{mL}$. Cardiotoxin injury was induced by injecting $20 \mu \mathrm{L}$ of a $10 \mu \mathrm{M}$ cardiotoxin solution in PBS into tibialis anterior or gastrocnemius/soleus muscles in sedated animals ( $3 \%$ isoflurane, $0.8 \mathrm{~L} / \mathrm{min} \mathrm{O}_{2}$ ) 2 hours after pretreatment. Cardiotoxin was released down the midline of the muscle to induce a homogeneous area of injury at the center of the muscle. Muscle was harvested 3 hours after cardiotoxin injection.

Evans blue dye uptake. Sections (10 $\mu \mathrm{m}$ thick) from the center of frozen-embedded muscles were collected on a cryostat (chamber, $-20^{\circ} \mathrm{C}$; sample, $-15^{\circ} \mathrm{C}$; catalog CM1950, Leica). Tissue sections were fixed with methanol for 2 minutes, rinsed, and mounted with Vectashield with DAPI (H-1200, Vector Laboratories). Imaging was performed using a Zeiss Axio Observer A1 microscope, using a $\times 10$ objective. ZEN software (Zeiss) was used for acquiring images. Fluorescence quantitation, surface plots, and muscle area calculations were performed using FIJI. For whole-tissue dye quantification, whole tissue was dissected, finely minced, weighed, and incubated at $55^{\circ} \mathrm{C}$ in $1 \mathrm{~mL}$ of formamide for 2 hours. Spectrophotometric absorbance was measured at $620 \mathrm{~nm}$.

Serum collection and CK analysis. Mice were sedated (3\% isoflurane, $0.8 \mathrm{~L} / \mathrm{min}_{2}$ ) and blood was collected by means of retro-orbital puncture with heparinized capillary tubes (20-362-566, Thermo Fisher Scientific) into Microtainer Gold Top Serum Separators (365967, Becton Dickinson) and centrifuged at $8,000 \mathrm{~g}$ for 10 minutes. The plasma fractions were frozen and stored at $-80^{\circ} \mathrm{C}$. Serum CK was analyzed in duplicate for each mouse using the EnzyChrom Creatine Kinase Assay (ECPK-100, BioAssay Systems) following the manufacturer's instructions and as described previously (33). Results were acquired with the Synergy HTX multi-mode plate reader (BioTek).

Short-term chronic dosing regimen. Sgcg-null mice were sedated (3\% isoflurane, $0.8 \mathrm{~L} / \mathrm{min}_{2}$ ) and blood collected as described above. While sedated, mice were injected with $1 \mathrm{mg} / \mathrm{kg}$ recombinant annexin A6 (5186-A6-050, R\&D Systems) or PBS into the right retro-orbital cavity once every 3 days for a total of 5 injections, and then blood drawn on day 14 after the initial injection. An additional cohort of $S g c g$-null mice had blood drawn as described above immediately prior to the first protein injection. Then, $1 \mathrm{mg} / \mathrm{kg}$ recombinant annexin A6 or annexin A2 (both produced by Northwestern's Protein Production Core) was injected into the right retro-orbital cavity of sedated mice, once every 3 days for a total of 5 injections and then blood drawn on day 14 after the initial injection.

Exercise injury. Sgcg-null mice were sedated (3\% isoflurane, 0.8 $\mathrm{L} / \mathrm{min} \mathrm{O}_{2}$ ) and blood was collected before exercise by means of left retro-orbital puncture as described above. While sedated, mice were then injected with $1 \mathrm{mg} / \mathrm{kg}$ recombinant annexin A6 (5186-A6-050, R\&D systems) or PBS plus $1 \mathrm{mg} / \mathrm{kg}$ BSA into the right retro-orbital cavity at 9 am and 5 pm for 5 consecutive injection over 48 hours. Two hours after the fifth retro-orbital injection, mice were subjected to 60 minutes of treadmill running at $10 \mathrm{~m} / \mathrm{min}$ at a $15^{\circ}$ decline. Thirty minutes after exercise, blood was collected from the left retro-orbital cavity. Serum CK was analyzed as described above. Injections, exercise, and blood draws were performed blinded to treatment group.

Protein production. Recombinant mouse annexin A6 and mouse annexin A2 were produced and purified by the Northwestern's recombinant protein production core. Briefly, mouse annexin A6 (MG222645, Origene) and annexin A2 (MG205064, Origene) were subcloned into the pCMV6-AC-His backbone (PS100002, Origene). Plasmids were transfected and expressed with an ExpiCHO expression system (A29133, Thermo Fisher Scientific). Carboxyl-terminal-tagged recombinant protein was purified with Ni-charged MagBeads (L00295, GenScript) and purity evaluated by SDS-PAGE. Protein purity was addition- 
ally validated by immunoblot using anti-His (MAB050, R\&D Systems) and anti-annexin A6 (ab31026, Abcam) or anti-annexin A2 (ab154113, Abcam) antibodies. Protein was diluted in PBS and stored at $-80^{\circ} \mathrm{C}$.

Calcium kinetics. FDB muscle was electroporated and isolated as described above. Myofibers were damaged in Ringer's solution with $\mathrm{Ca}^{2+}$ concentrations of $2 \mathrm{mM}, 1 \mathrm{mM}, 0.5 \mathrm{mM}, 0.25 \mathrm{mM}, 0.175 \mathrm{mM}$, $0.1 \mathrm{mM}, 0.050 \mathrm{mM}$, and $0 \mathrm{mM}$. EDTA was added as a $\mathrm{Ca}^{2+}$-chelating agent in only in the $0 \mathrm{mM} \mathrm{Ca}^{2+}$ Ringer's. Myofibers were isolated directly into $2 \mathrm{mM}, 1 \mathrm{mM}$, and $0.5 \mathrm{mM}$ Ringer's for those experiments, respectively. For experiments using less than $0.5 \mathrm{mM} \mathrm{Ca}^{2+}$, myofibers were isolated in $0.5 \mathrm{mM} \mathrm{Ca}^{2+}$ Ringer's and then diluted with $0 \mathrm{mM}$ EDTA-free $\mathrm{Ca}^{2+}$ Ringer's. For the $0 \mathrm{mM}$ experiments, myofibers were isolated in $0.5 \mathrm{mM} \mathrm{Ca}^{2+}$ Ringer's, which was then replaced with $0 \mathrm{mM}$ $\mathrm{Ca}^{2+}$ Ringer's with EDTA just prior to imaging. Coelectroporation of wild-type annexin and wild-type annexin constructs was performed in one mouse foot, while the contralateral foot was coelectroporated with wild-type annexin and mutant annexin. All measurements were acquired from myofibers isolated from at least $n=2$ mice, $n=3$ myofibers per mouse at each $\mathrm{Ca}^{2+}$ concentration. Repair cap areas were fitted with a Hill curve at $\mathrm{Ca}^{2+}$ concentrations ranging from $0-2 \mathrm{mM}$. Kinetic parameters were calculated using Prism (GraphPad).

Calcium and $\mathrm{pH}$ indicator dye measurements. Wild-type FDBs were isolated and plated in Ringer's on Matek glass-bottom dishes as described above. Twenty minutes prior to imaging, myofibers were loaded with Fluo-4 AM at $37^{\circ} \mathrm{C}$ (F10489, Thermo Fisher Scientific) or pHrodo AM (P35373, Thermo Fisher Scientific) as described in the instruction manual. Fibers were rinsed once with Ringer's and then subsequently damaged and imaged on the Nikon A1R GaSP, as described above. Fluorescence intensity was measured using FIJI. pHrodo change in fluorescence intensity was calculated as F/FO. Data were acquired from $n=3$ mice per experiment from multiple myofibers per mouse. Additionally, wild-type myofibers were incubated in $0 \mathrm{mM} \mathrm{Ca}^{2+}$ for 1 hour, preloaded with Fluo-4 AM for 20 minutes prior to imaging, rinsed, and then damaged on the Nikon A1R-MP+ in the presence of $0 \mathrm{mM}$ external $\mathrm{Ca}^{2+}$ with or without EGTA in the external Ringer's solution.

Cardiotoxin injection and histology. Wild-type mice were sedated ( $3 \%$ isoflurane, $0.8 \mathrm{~L} / \mathrm{min} \mathrm{O}_{2}$ ) and then injected with $1 \mathrm{mg} / \mathrm{kg}$ recombinant annexin A6 (5186-A6-050, R\&D Systems) or PBS plus $1 \mathrm{mg} / \mathrm{kg}$ BSA into the right retro-orbital cavity and allowed to recover. Two hours after injection mice were sedated for a second time. While sedated, the tibialis anterior muscles were injected along the midline with $10 \mu \mathrm{M}$ cardiotoxin in $20 \mu \mathrm{L}$ of PBS as described above. Seven days after injection, the muscle was isolated and frozen. Muscle sections were acquired every $100 \mu \mathrm{m}$ from muscle tendon into the mid belly, fixed, and stained with hematoxylin and eosin. Imaging was performed using a Zeiss Axio Observer A1 microscope with a $\times 10$ objective. ZEN software (Zeiss) was used for acquiring tiled images. Percentage injury area was calculated as the average injured area (containing internal myonuclei) divided by total muscle area of 3 sections per muscle. One muscle from each group was excluded due to a technical error in tissue processing. Damage area was measured using FIJI.
Single-cell $\mathrm{Ca}^{2+}$ and shortening measurements. Isolated FDB fibers were plated on laminin-coated, glass-bottomed, 35-mm dishes for 1 hour and then cultured overnight in DMEM with $10 \%$ FBS and $1 \%$ penicillin/streptomycin at $37^{\circ} \mathrm{C}$ in a $10 \% \mathrm{CO}_{2}$ incubator. One hour prior to data acquisition, the medium was removed and cells were incubated in Tyrode buffer (119 mM NaCl, $5 \mathrm{mM} \mathrm{KCl,} 25 \mathrm{mM}$ HEPES, $2 \mathrm{mM} \mathrm{CaCl}_{2}$, and $2 \mathrm{mM} \mathrm{MgCl}_{2}$ ) with $10 \mu \mathrm{M}$ Indo- $1 \mathrm{AM}$ (TefLabs) for 1 hour at $37^{\circ} \mathrm{C}$ in a $10 \% \mathrm{CO}_{2}$ incubator. Dishes were then filled with Tyrode buffer, mounted on a custom stage, and platinum pacing electrodes were inserted into the dish. Stimulation was elicited using a 701C high-powered stimulator controlled by the 950A software (Aurora Scientific). Stimulation was performed at 40 and $80 \mathrm{~Hz}, 5-\mathrm{ms}$ pulse width, 100-ms duration. Ratiometric $\mathrm{Ca}^{2+}$ signals were collected with 2 photomultiplier tubes and a FluoroDaq controller. Video sarcomere length was recorded with a highspeed camera and fast Fourier transform using the Aurora Scientific 900B-VSL system. Ten transients were collected over 20 seconds and averaged together per cell per frequency.

Statistics. Statistical analyses were performed with Prism (Graph$\mathrm{Pad}$ ). Comparisons relied on ANOVA (1-way ANOVA for 1 variable, 2-way ANOVA for 2 variables; typically area and $\mathrm{Ca}^{2+}$ concentration). Otherwise, unpaired 2-tailed $t$ tests were performed. $P$ values of less than or equal to 0.05 were considered significant. Error bars represent \pm standard error of the mean (SEM).

Study approval. The study was conducted with the approval of Northwestern University's Institutional Animal Care and Use Committee.

\section{Author contributions}

ARD, JLW, and EB contributed to myofiber preparations and acquisition of data. KSF and CCO prepared reagents and performed imaging. ARD, KF, and CCO performed the studies on recombinant annexin A6. DYB and JJS performed the baseline $\mathrm{Ca}^{2+}$ experiments. PGP performed the histological staining. MQ made critical contributions to the manuscript preparation. ARD and EMM designed the studies, analyzed the data, and wrote the manuscript.

\section{Acknowledgments}

This work was supported by NIH grants NS047726, AR052646, HL61322, HL141698, Muscular Dystrophy Association Development grant 479350, and Parent Project Muscular Dystrophy. We acknowledge the outstanding support Constadina Arvanitis at the Center for Advanced Microscopy at Northwestern University. Additionally, we acknowledge the Recombinant Protein Production Core at Northwestern University.

Address correspondence to: Alexis R. Demonbreun or Elizabeth M. McNally, Center for Genetic Medicine, Northwestern University, 303 E Superior Lurie 5-512 (ARD) or 5-516 (EMM), Chicago, Illinois 60611, USA. Phone: 312.503.6249; Email: alexis.demonbreun@ northwestern.edu (ARD). Phone: 312.503.5600; Email: elizabeth. monally@northwestern.edu (EMM).
1. McNeil PL, Khakee R. Disruptions of muscle fiber plasma membranes. Role in exercise-induced damage. Am J Pathol. 1992;140(5):1097-1109.

2. McNeil PL, Kirchhausen T. An emergency response team for membrane repair. Nat Rev Mol Cell Biol. 2005;6(6):499-505.

3. Rodríguez A, Webster P, Ortego J, Andrews NW. Lysosomes behave as $\mathrm{Ca}^{2+}$-regulated exocytic vesicles in fibroblasts and epithelial cells. J Cell Biol. 1997;137(1):93-104.

4. Reddy A, Caler EV, Andrews NW. Plasma membrane repair is mediated by $\mathrm{Ca}(2+)$-regulated exo- 
cytosis of lysosomes. Cell. 2001;106(2):157-169.

5. Demonbreun AR, Quattrocelli M, Barefield DY, Allen MV, Swanson KE, McNally EM. An actin-dependent annexin complex mediates plasma membrane repair in muscle. JCell Biol. 2016;213(6):705-718.

6. McDade JR, Archambeau A, Michele DE. Rapid actin-cytoskeleton-dependent recruitment of plasma membrane-derived dysferlin at wounds is critical for muscle membrane repair. FASEB J 2014;28(8):3660-3670.

7. Babiychuk EB, Monastyrskaya K, Potez S, Draeger A. Blebbing confers resistance against cell lysis. Cell Death Differ. 2011;18(1):80-89.

8. Bansal D, et al. Defective membrane repair in dysferlin-deficient muscular dystrophy. Nature. 2003;423(6936):168-172.

9. Bashir R, et al. A gene related to Caenorhabditis elegans spermatogenesis factor fer- 1 is mutated in limb-girdle muscular dystrophy type 2B. Nat Genet. 1998;20(1):37-42.

10. Demonbreun AR, et al. Eps 15 homology domain (EHD)-1 remodels transverse tubules in skeletal muscle. PLoS One. 2015;10(9):e0136679.

11. Demonbreun AR, McNally EM. Plasma membrane repair in health and disease. Curr Top Membr. 2016;77:67-96.

12. Defour A, et al. Annexin A2 links poor myofiber repair with inflammation and adipogenic replacement of the injured muscle. Hum Mol Genet. 2017;26(11):1979-1991.

13. Cai C, et al. MG53 nucleates assembly of cell membrane repair machinery. Nat Cell Biol. 2009;11(1):56-64.

14. Jimenez AJ, Perez F. Plasma membrane repair: the adaptable cell life-insurance. Curr Opin Cell Biol. 2017;47:99-107.

15. Lauritzen SP, Boye TL, Nylandsted J. Annexins are instrumental for efficient plasma membrane repair in cancer cells. Semin Cell Dev Biol. 2015;45:32-38.

16. Bizzarro V, Petrella A, Parente L. Annexin A1: novel roles in skeletal muscle biology. J Cell Physiol. 2012;227(8):3007-3015

17. Grewal T, et al. Annexin A6-A multifunctional scaffold in cell motility. Cell Adh Migr. 2017;11(3):288-304

18. Boye TL, et al. Annexins induce curvature on free-edge membranes displaying distinct morphologies. Sci Rep. 2018;8(1):10309.

19. Boye TL, et al. Annexin A4 and A6 induce membrane curvature and constriction during cell membrane repair. Nat Commun. 2017;8(1):1623.

20. Blackwood RA, Ernst JD. Characterization of Ca2(+)-dependent phospholipid binding, vesicle aggregation and membrane fusion by annexins. Biochem J.1990;266(1):195-200.

21. Zaks WJ, Creutz CE. Ca(2+)-dependent annexin self-association on membrane surfaces. Biochemistry. 1991;30(40):9607-9615.

22. Benz J, et al. The structure of recombinant human annexin VI in crystals and membrane-bound. JMol Biol. 1996;260(5):638-643.

23. Buzhynskyy N, et al. Annexin-A6 presents two modes of association with phospholipid membranes. A combined QCM-D, AFM and cryoTEM study. J Struct Biol. 2009;168(1):107-116.

24. Gerke V, Creutz CE, Moss SE. Annexins: linking
$\mathrm{Ca}^{2+}$ signalling to membrane dynamics. Nat Rev Mol Cell Biol. 2005;6(6):449-461.

25. Fiehn W, Peter JB, Mead JF, Gan-Elepano M. Lipids and fatty acids of sarcolemma, sarcoplasmic reticulum, and mitochondria from rat skeletal muscle. J Biol Chem. 1971;246(18):5617-5620.

26. Babbin BA, et al. Annexin A1 regulates intestinal mucosal injury, inflammation, and repair. JImmunol. 2008;181(7):5035-5044.

27. Lennon NJ, Kho A, Bacskai BJ, Perlmutter SL, Hyman BT, Brown RH. Dysferlin interacts with annexins $\mathrm{A} 1$ and $\mathrm{A} 2$ and mediates sarcolemmal wound-healing. J Biol Chem. 2003;278(50):50466-50473.

28. McNeil AK, Rescher U, Gerke V, McNeil PL. Requirement for annexin $\mathrm{A} 1$ in plasma membrane repair.J Biol Chem. 2006;281(46):35202-35207.

29. Roostalu U, Strähle U. In vivo imaging of molecular interactions at damaged sarcolemma. Dev Cell. 2012;22(3):515-529.

30. Davenport NR, Sonnemann KJ, Eliceiri KW, Bement WM. Membrane dynamics during cellular wound repair. Mol Biol Cell.2016;27(14):2272-2285

31. Carmeille R, et al. Annexin-A5 promotes membrane resealing in human trophoblasts. Biochim Biophys Acta. 2015;1853(9):2033-2044.

32. Bement WM, Mandato CA, Kirsch MN. Wound-induced assembly and closure of an actomyosin purse string in Xenopus oocytes. Curr Biol. 1999;9(11):579-587.

33. Demonbreun AR, et al. Enhanced muscular dystrophy from loss of dysferlin is accompanied by impaired annexin $\mathrm{A} 6$ translocation after sarcolemmal disruption. Am J Pathol. 2016;186(6):1610-1622.

34. Quattrocelli M, et al. Genetic modifiers of muscular dystrophy act on sarcolemmal resealing and recovery from injury. PLoS Genet. 2017;13(10): 1007070.

35. Swaggart KA, et al. Annexin A6 modifies muscular dystrophy by mediating sarcolemmal repair. Proc Natl Acad Sci USA. 2014;111(16):6004-6009.

36. Quattrocelli M, et al. Intermittent glucocorticoid steroid dosing enhances muscle repair without eliciting muscle atrophy. J Clin Invest. 2017;127(6):2418-2432.

37. Akerboom J, et al. Optimization of a GCaMP calcium indicator for neural activity imaging. J Neurosci. 2012;32(40):13819-13840.

38. Demonbreun AR, McNally EM. DNA electroporation, isolation and imaging of myofibers. J Vis Exp. 2015;(106):e53551.

39. Gerke V, Moss SE. Annexins: from structure to function. Physiol Rev. 2002;82(2):331-371.

40. Bi GQ, Alderton JM, Steinhardt RA. Calcium-reg ulated exocytosis is required for cell membrane resealing. JCell Biol. 1995;131(6 Pt 2):1747-1758.

41. Yeung T, et al. Contribution of phosphatidylserine to membrane surface charge and protein targeting during phagosome maturation. J Cell Biol. 2009;185(5):917-928.

42. Zweifach A. FM1-43 reports plasma membrane phospholipid scrambling in T-lymphocytes. Biochem J. 2000;349(Pt 1):255-260.

43. Jost M, Thiel C, Weber K, Gerke V. Mapping of three unique $\mathrm{Ca}(2+)$-binding sites in human annexin II. Eur J Biochem. 1992;207(3):923-930.

44. Avila-Sakar AJ, Creutz CE, Kretsinger RH.
Crystal structure of bovine annexin VI in a calcium-bound state. Biochim Biophys Acta. 1998;1387(1-2):103-116.

45. Jennische E, Hansson HA. Postischemic skeletal muscle injury: patterns of injury in relation to adequacy of reperfusion. Exp Mol Pathol. 1986;44(3):272-280.

46. Hack AA, Cordier L, Shoturma DI, Lam MY, Sweeney HL, McNally EM. Muscle degeneration without mechanical injury in sarcoglycan deficiency. Proc Natl Acad Sci USA. 1999;96(19):10723-10728.

47. Millay DP, Goonasekera SA, Sargent MA, Maillet $\mathrm{M}$, Aronow BJ, Molkentin JD. Calcium influx is sufficient to induce muscular dystrophy through a TRPC-dependent mechanism. Proc Natl Acad Sci USA. 2009;106(45):19023-19028.

48. Goonasekera SA, et al. Mitigation of muscular dystrophy in mice by SERCA overexpression in skeletal muscle. JClin Invest. 2011;121(3):1044-1052.

49. Demonbreun AR, et al. Dysferlin and myoferlin regulate transverse tubule formation and glycerol sensitivity. Am J Pathol. 2014;184(1):248-259.

50. Murphy S, et al. Proteomic analysis of the sarcolemma-enriched fraction from dystrophic $\mathrm{mdx}-4 \mathrm{cv}$ skeletal muscle. J Proteomics. 2019;191:212-227.

51. La M, et al. Annexin 1 peptides protect against experimental myocardial ischemia-reperfusion: analysis of their mechanism of action. FASEB J. 2001;15(12):2247-2256.

52. Meng H, Zhang Y, An ST, Chen Y. Annexin A3 gene silencing promotes myocardial cell repair through activation of the PI3K/Akt signaling pathway in rats with acute myocardial infarction. JCell Physiol. 2019;234(7):10535-10546.

53. Quattrocelli M, Salamone IM, Page PG, Warner JL, Demonbreun AR, McNally EM. Intermittent glucocorticoid dosing improves muscle repair and function in mice with limb-girdle muscular dystrophy. Am J Pathol. 2017;187(11):2520-2535

54. Waddell LB, et al. Dysferlin, annexin A1, and mitsugumin 53 are upregulated in muscular dystrophy and localize to longitudinal tubules of the T-system with stretch. J Neuropathol Exp Neurol. 2011;70(4):302-313.

55. Duann P, et al. MG53-mediated cell membrane repair protects against acute kidney injury. $S c i$ Transl Med.2015;7(279):279ra36.

56. He B, et al. Enhancing muscle membrane repair by gene delivery of MG53 ameliorates muscular dystrophy and heart failure in $\delta$-sarcoglycan-deficient hamsters. Mol Ther. 2012;20(4):727-735.

57. Jia Y, et al. Treatment of acute lung injury by targeting MG53-mediated cell membrane repair. Nat Commun. 2014;5:4387.

58. Liu J, et al. Cardioprotection of recombinant human MG53 protein in a porcine model of ischemia and reperfusion injury. J Mol Cell Cardiol. 2015;80:10-19.

59. Weisleder N, et al. Recombinant MG53 protein modulates therapeutic cell membrane repair in treatment of muscular dystrophy. Sci Transl Med. 2012;4(139):139ra85.

60. Ceco E, et al. Targeting latent TGF $\beta$ release in muscular dystrophy. Sci Transl Med. 2014;6(259):259ra144.

61. DiFranco M, Quinonez M, Capote J, Vergara J. DNA transfection of mammalian skeletal muscles using in vivo electroporation. JVis Exp. 2009;(32):1520. 